\title{
Analysis of partial trade credit financing in a supply chain by EOQ-based model for decaying items with shortages
}

\author{
Karuppuchamy Annadurai • Ramasamy Uthayakumar
}

Received: 29 July 2010 / Accepted: 8 November 2011 / Published online: 11 December 2011

(C) The Author(s) 2011. This article is published with open access at Springerlink.com

\begin{abstract}
By allowing shortages as backlogging, the impact on the cost from the decay of the products can be balanced out. To attract more sales, suppliers frequently offer a trade credit if the retailer orders more than or equal to a predetermined quantity. In this study, we analyze the partial trade credit financing in a supply chain by economic order quantity-based model for decaying items including shortages. We assume that the supplier may offer a partial permissible delay in payments even if the order quantity is less than predetermined quantity. Lemmas and theorems to determine the criterion for the existence and uniqueness of the minimum solution is subsequently developed. A computer code using the software Matlab 7.0 is developed to derive the optimal solution, and a numerical example is presented to illustrate the procedures of algorithm. The results in the numerical example indicate that the retailer trades off the benefits of full delay in payments against the partial delay in payments and always enjoys the full delay in payments. Finally, some important managerial insights are also inferred from the sensitivity analysis of the optimal solution with respect to the major parameters of the system.
\end{abstract}

\footnotetext{
K. Annadurai $(\bowtie)$

Department of Mathematics, Government Arts College

(Autonomous), Karur 639005, Tamil Nadu, India

e-mail: anna.vadivu@gmail.com

R. Uthayakumar

Department of Mathematics, Gandhigram Rural University,

Gandhigram 624302, Tamil Nadu, India
}

Keywords Inventory $\cdot$ Partial trade credit . Deterioration $\cdot$ Shortage $\cdot$ Optimization

\section{Introduction}

In recent years, most researches in the area of inventory control have been oriented toward the development of more realistic and practical models for decision makers. Both in deterministic and probabilistic inventory models of classical type, it is observed that payments are made to the supplier immediately after receiving the items. In practice, the supplier will offer the retailer a delay period in paying for the amount of purchase to increase the demand known as trade credit period. Offering such a credit period to the retailer will encourage the supplier's selling and reduce on-hand stock level. Simultaneously, without a primary payment, the retailer can take the advantages of a credit period to reduce cost and increase profit. Thus, the delay in the payment offered by the supplier is a kind of price discount since paying later indirectly reduces the cost of holding, and it encourages the retailer to increase their order quantity. Moreover, during this credit period, the retailer can start to accumulate revenues on the sales and earn interest on that revenue. Hence, paying later indirectly reduces the cost of holding stock. But a higher interest is charged if the payment is not settled by end of this credit period. Hence, trade credit can play an important role in inventory model for both the suppliers as well as the retailers.

In recent two decades, the effect of a permissible delay in payments on the optimal inventory system has received much attention from numerous researchers. Teng [1] indicated that the trade credit produces two 
benefits to the supplier: (1) It should attract new customers who consider it to be a type of price reduction, and (2) it should cause a reduction in sales outstanding, since some established customers will pay more promptly in order to take advantage of trade credit more frequently. Over the years, a number of researchers have appeared in the literature that treat inventory problems with varying conditions under trade credit intended to link financing, marketing, as well as operations concerns. Some of the prominent papers are discussed below.

Haley and Higgins [2] introduced the first inventory model to consider the economic order quantity (EOQ) under conditions of permissible delay in payments with deterministic demand, no shortages, and zero lead time. After that, numerous studies dealing with the trade credit problem have been presented. Hwang and Shinn [3] considered the problem of determining the retailer's optimal price and lot size simultaneously when the suppliers permit delay in payments. Jamal et al. [4] studied a wholesaler-retailer supply chain where the retailer is given a permissible credit period to pay back the dues without paying any interest to the wholesaler. Goyal [5] considered a model similar to that of Haley and Higgins [1] model with the exclusion of the penalty cost due to a late payment. Abbad and Jaggi [6] developed a sellerbuyer model with the permissible delay in payments by game theory to determine the optimal unit price under credit period, considering that the demand rate is a function of retail price. Huang [7] presented an inventory model assuming that the retailer also prefers a credit period to his customers which is shorter than the credit period offered by the supplier, in order to stimulate the demand. Chung et al. [8] developed optimal inventory policies under permissible delay in payments depending on the ordering quantity. Teng et al. [9] developed the optimal pricing and lot sizing under permissible delay in payments by considering the difference between the selling price and the purchase cost, and demand is a function of price. Xiping Song and Xiaoqiang [10] discussed on optimal payment time for a retailer under permitted delay of payment by the wholesaler. Goyal et al. [11] established an economic ordering quantity model for a retailer when the supplier offers a progressive interest charge and provided an easy-to-use closed form solution to the problem.

It is important to control and maintain the inventories of deteriorating items for the modern corporation. In daily life, the deteriorating of goods is a common phenomenon. Recently, deteriorating of item in inventory system has become an interesting topic due to its practical importance. Deterioration refers to decay, damage, or spoilage. In respect of foods, films, drugs, chemicals, electronic components, and radioactive substances, deterioration may happen during normal period of storage. Therefore, the loss due to deterioration cannot be neglected. Deteriorating inventory models have been widely studied in recent years. There are some research articles dealing with deteriorating inventory model under trade credit. Agarwal and Jaggi [12] developed ordering policies of deteriorating items under permissible delay in payments. Chang et al. [13] established an inventory model for deteriorating items with linear trend demand under conditions of permissible delay in payments. Dye and Chung [14] proposed inventory model by considering the stock-dependent demand for deteriorating items for partial backlogging and conditions of permissible delay in payments. They assume initial stock-dependent demand function. In a recent paper, Ouyang et al. [15] derived optimal ordering policy for deteriorating items with partial backlogging under permissible delay in payments. In the existing literature about permissible delay in payments, it is assumed that the demand is either mostly a function of time or a function of the retail price. Tsao and Sheen [16] studied the problem of dynamic pricing, promotion, and replenishment for a deteriorating item subject to the supplier's trade credit and retailer's promotional effort. In all the above articles, although the presence of credit period has been incorporated in the mathematical models, the impact of credit period on demand is ignored. Jaggi et al. [17] investigated the impact of credit linked demand on the retailer's optimal replenishment policy. In a recent paper, Soni and Shah [18] established optimal ordering policy for stock-dependent demand under progressive payment scheme.

In real life, the occurrence of shortage in an inventory system is phenomenon. Under most market behaviors, we can often observe that many products of famous brands or fashionable goods such as certain brand gum shoes, hi-fi equipment, and clothes may lead to a situation in which customers may prefer to wait for backorders while shortages occur. Besides the product, the image of selling shop is one of the potential factors that will motivate the customers intention of backorders. In many real situations, for the fashionable commodities and high-tech products with short product life cycle, the willingness for a customer to wait for backlogging is diminishing with the length of the waiting time. Hence, during a shortage period, the longer the waiting time is, the smaller the backlogging would be. Permitting limited planned shortages can reduce the pressure on high production capacity and 
hence result in a smother production schedule. Firms are able to maintain a backlog of orders to certain loyal customers without losing their business. However, the costs of shortages or lost sales should not be exorbitant to facilitate the feasibility of the strategy. If the cost of holding inventory is significantly higher than the shortage cost, permitting occasional brief shortages to lower the average inventory level may be a sound business practice to reduce the total cost. To reflect this phenomenon, Teng et al. [19] developed inventory models with shortages. Yang et al. [20] proposed deterministic inventory lot size models under inflation with shortages and deterioration for fluctuating demand. Most researchers assumed that shortages are completely backlogged. In practice, some customers would like to wait for backlogging during the shortage period, but the others would not. Consequently, the opportunity cost due to lost sales should be considered in the modeling. Papachristos and Skouri [21] proposed a partially backlog inventory model in which the backlogging rate decreases exponentially as the waiting time increases. Teng et al. [22] established inventory models for deteriorating items with time varying demand and partial backlogging.

To attract more sales, suppliers often offer a permissible delay in payments if the retailer orders more than or equal to a predetermined quantity. On the other hand, the supplier may offer a partial permissible delay in payments even if the order quantity is less than predetermined quantity and such a system is termed as partial trade credit. In supply chain management, partial trade credit financing is one of central features. In practice, this partial trade credit financing at a retailer is more matched to real-life situations in a supply chain. Recently, Ouyang et al. [23] developed an economic order quantity model for deteriorating items with partially permissible delay in payments linked to order quantity. In their model, it is assumed that shortage is not allowed.

In this study, we extend Ouyang et al. [23] model by allowing shortages and analyze the partial trade credit financing in a supply chain by EOQ-based model for decaying items. We assume that the supplier may offer a partial permissible delay in payments even if the order quantity is less than predetermined quantity. The goal of this research is to determine the optimal ordering policy in order to minimize the total relevant cost. The remainder of this paper is organized as follows: Section 2 describes the notations and assumptions employed throughout this paper. We formulate the proposed model mathematically in Section 3. In Section 4, several theoretical results by means of eight lemmas and three theorems are established. Following this, an efficient algorithm is designed to determine the optimal policy. Section 5 shows that the inventory models of numerous previous works are special cases of this study. To highlight and visualize the results, a specific example is then given in Section 6. In Section 7, sensitivity analysis is carried out and some managerial insights are obtained. Finally, we draw some conclusions and provide some suggestions for future research in Section 8 .

\section{Notations and assumptions}

Notations and assumptions from Ouyang et al. [23] model are adopted except the backlogging parameter, shortage cost for backlogged item, and unit selling price of the item. We outline these notations below for the sake of completeness and easy reference.

\subsection{Notations}

The notation is summarized in the following:

$A$ ordering cost per order

$Q \quad$ ordering quantity

$D$ annual demand

$w$ the quantity at which the full delay payment permitted per order

$t_{w} \quad$ the time interval that $w$ units are depleted to zero

$\theta \quad$ the deterioration rate, where $0 \leq \theta<1$

$\delta \quad$ backlogging parameter, where $0 \leq \delta(t) \leq 1$

$C$ unit purchase cost of the item

$C_{1} \quad$ shortage cost for backlogged item

$C_{2} \quad$ unit cost of lost sales

$h \quad$ holding cost per unit per unit time excluding interest charges

$P \quad$ unit selling price of the item

$M \quad$ the permissible delay period offered by the supplier for settling the accounts to the retailer

$\alpha \quad$ the fraction of the delay payments permitted by the supplier per order, $0 \leq \alpha \leq 1$

$I_{\mathrm{e}} \quad$ interest which can be earned per dollar per unit time

$I_{\mathrm{p}} \quad$ interest payable per dollar in stocks per unit time

$T$ length of order cycle

$t_{1} \quad$ length of time in which the inventory has no shortage

$I(t)$ inventory level at time $t$ 


\subsection{Assumptions}

The following assumptions are made in the model:

1. The system involves single item.

2. Replenishment occurs instantaneously at an infinite rate.

3. Lead time is negligible.

4. Demand rate is known with certainty and uniform.

5. Shortages are allowed and partially backordered, i.e., only a fraction of shortages backordered is a differentiable and decreasing function of time $t$ denoted by $\delta(t)$, where $t$ is the waiting time up to the next replenishment with $0 \leq \delta(t) \leq 1$. Let $B(t)$ denote this fraction and is given by $B(t)=\frac{1}{1+\delta t}$.

6. There is no repair or replacement of deteriorated units during the planning horizon. The item will be withdrawn from warehouse immediately as they become deteriorated.

7. The inventory carrying cost and deterioration cost are assumed proportional to the inventory level and incur instantaneously.

8. During the trade credit period $M$, the account is not settled, and generated sales revenue is deposited in an interest bearing account. At the end of the period, the retailer pays off all units bought and starts to pay the capital opportunity cost for the items in stock.

9. If the retailer orders more than or equal to a predetermined quantity, then he has a grace period to make the full payment. Otherwise, he must pay the payment for goods of certain proportion first while receiving the goods and has a grace period to pay off the rest. In such situation, the retailer must take a loan with the interest charged $I_{\mathrm{p}}$ to pay the supplier the partial payment $(1-\alpha) C Q$ when the order is filled at time 0 .

10. Total cost per unit time is given by $\mathrm{TC}\left(t_{1}, T\right)=$ ordering cost + holding cost (excluding interest charges) + shortage cost due to backlogging + opportunity cost due to lost sales + cost of interest charges for the unsold items after the permissible delay in payments - interest earned from the sales during the permissible period.

\section{Mathematical formulation}

In this section, a mathematical model is developed to determine the optimal replenishment cycle time that minimizes the total annual relevant cost in an inventory system for deteriorating items under partial permissible delay in payments including shortages. Due to both the

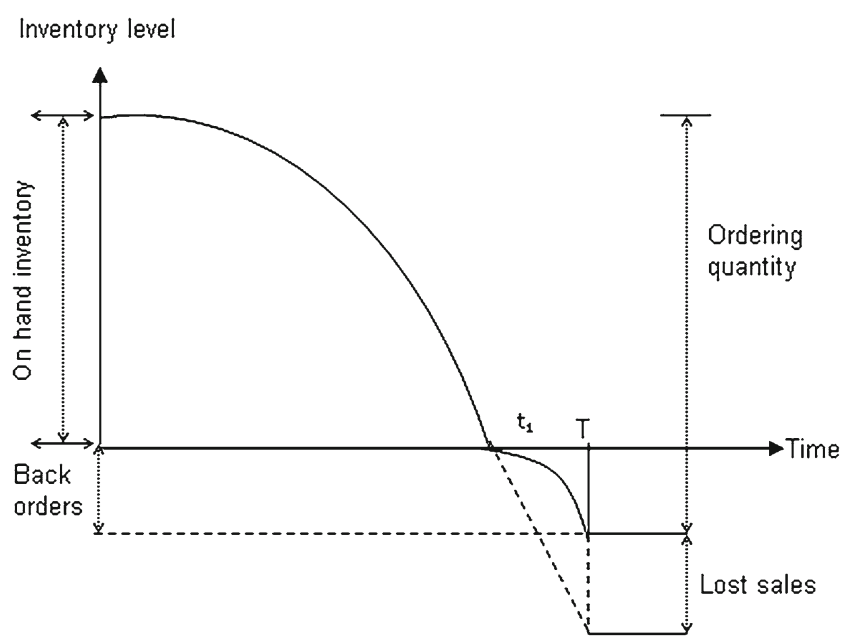

Fig. 1 Graphical representation of the inventory system

demand and deterioration of the item, the inventory level decreases during the period $\left[0, t_{1}\right]$ and ultimately falls to zero at $t=t_{1}$. Thereafter, shortages are allowed to occur and the demand during the period $\left[t_{1}, T\right]$ is partially backlogged. The behavior of inventory system at any time is depicted in Fig. 1.

As described above, the inventory level decreases owing to demand as well as deterioration during the time interval $\left[0, t_{1}\right]$. Hence, the differential equation representing the inventory status is given by

$\frac{d I_{1}(t)}{d t}+\theta I_{1}(t)=-D ; \quad 0 \leq t \leq t_{1}$,

with boundary condition $I_{1}(0)=I_{\max }$. The solution of Eq. 1 is given by

$I_{1}(t)=\frac{D}{\theta}\left[e^{\theta\left(t_{1}-t\right)}-1\right]$ if $0 \leq t \leq t_{1}$.

So, the maximum inventory level for each cycle can be obtained as

$I_{\max }=I_{1}(0)=\frac{D}{\theta}\left(e^{\theta t_{1}}-1\right)$.

During the shortage interval $\left[t_{1}, T\right]$, the demand at time $t$ is partially backlogged at the fraction $\frac{1}{1+\delta(T-t)}$. Thus, the differential equation governing the amount of demand backlogged is as below.

$\frac{d I_{2}(t)}{d t}=-\frac{D}{1+\delta(T-t)} ; \quad t_{1} \leq t \leq T$,

with boundary condition $I_{2}\left(t_{1}\right)=0$. The solution of Eq. 4 is given by

$$
\begin{aligned}
I_{2}(t)= & \frac{D}{\delta}\left\{\ln [1+\delta(T-t)]-\ln \left[1+\delta\left(T-t_{1}\right)\right]\right\} \\
& \text { if } t_{1} \leq t \leq T .
\end{aligned}
$$


Thus, we have

$I(t)= \begin{cases}\frac{D}{\theta}\left[e^{\theta\left(t_{1}-t\right)}-1\right] & \text { if } 0 \leq t \leq t_{1} \\ \frac{D}{\delta}\left[\ln [1+\delta(T-t)]-\ln \left[1+\delta\left(T-t_{1}\right)\right]\right] & \text { if } t_{1} \leq t \leq T .\end{cases}$

By letting $t=T$ in Eq. 5, we can obtain the maximum amount of demand backlogged per cycle as

$S=-I_{2}(T)=\frac{D}{\delta} \ln \left[1+\delta\left(T-t_{1}\right)\right]$.

Hence, the order quantity per cycle is given by

$Q=I_{\max }+S=\frac{D}{\theta}\left(e^{\theta t_{1}}-1\right)+\frac{D}{\delta} \ln \left[1+\delta\left(T-t_{1}\right)\right]$.

From Eq. 8, we can obtain the time interval that $w$ units are depleted to zero due to both demand and deterioration as $t_{w}=\frac{1}{\theta} \ln \left(\frac{\theta w}{D}+1\right)$.

If the retailer orders more than or equal to a predetermined quantity, then he has a grace period to make the full payment. Otherwise, he must pay the payment for goods of certain proportion first while receiving the goods and has a grace period to pay off the rest. Consequently, it is easy to see that the inequality $Q \geq w$ holds iff $t_{1} \geq t_{w}$. If $Q \geq w$ (i.e., $t_{1} \geq t_{w}$ ), then fully delayed payment is permitted. On the other hand, if the retailer orders less than a predetermined quantity, then he has only a partial delay in payments. In such situation, the retailer must take a loan with the interest to pay the supplier the partial payment. Hence, if $Q<w$ (i.e., $t_{1}<$ $t_{w}$ ), then the retailer must take a loan with the interest charged $I_{\mathrm{p}}$ to pay the supplier the partial payment $(1-\alpha) C Q$ when the order is filled at time 0 and then pays the rest on the last time of the credit period. From the constant sales revenue $P D$, the retailer will be able to payoff the loan $(1-\alpha) C Q$ at time $\frac{(1-\alpha) C}{P}\left(\frac{e^{\theta t_{1}}-1}{\theta}\right)$. Also if it is shorter or equal to the permissible delay $M$, then $t_{1} \leq t_{0}=\frac{1}{\theta} \ln \left[\frac{\theta P M}{(1-\alpha) C}+1\right]$ and vice versa. Here, it is noted that $t_{0}>M$.

Now, the total annual cost of the inventory system for the retailer is computed using the following various components:

- $\quad$ Annual ordering cost $=\frac{A}{T}$

- Excluding interest charges, the annual stock holding cost $=\frac{h}{T} \int_{0}^{t_{1}} I_{1}(t) d t=\frac{h D}{\theta^{2} T}\left[e^{\theta t_{1}}-\theta t_{1}-1\right]$
- Annual deterioration cost $=\frac{C \theta}{T} \int_{0}^{t_{1}} I_{1}(t) d t=$ $\frac{C D}{\theta T}\left[e^{\theta t_{1}}-\theta t_{1}-1\right]$

- Shortage cost due to backlogging $=\frac{C_{1}}{T} \int_{t_{1}}^{T}-$ $I_{2}(t) d t=\frac{C_{1} D}{\delta T}\left[\left(T-t_{1}\right)-\frac{\ln \left[1+\delta\left(T-t_{1}\right)\right]}{\delta}\right]$

- $\quad$ Opportunity cost due to lost sales $=\frac{C_{2}}{T} \int_{t_{1}}^{T} D[1-$ $B(T-t)] d t=\frac{C_{2} D}{T}\left[\left(T-t_{1}\right)-\frac{\ln \left[1+\delta\left(T-t_{1}\right)\right]}{\delta}\right]$

Regarding the interest charged and interest earned, based on the values of $M, t_{w}$, and $t_{0}$, we have the following three possible cases and we discuss each case in detail:

(I) $t_{0}>M \geq t_{w}$, (II) $t_{0} \geq t_{w}>M$, and (III) $t_{w}>t_{0}>M$.

Case I $t_{0}>M \geq t_{w}$

In this case, there are three sub-cases.

Case $i \quad M \leq t_{1}$

When the end point of credit period is smaller than or equal to the length of period with positive inventory stock of the item $\left(M \leq t_{1}\right)$, payment of goods is settled and the retailer starts paying the capital opportunity cost for the items in stock with rate $I_{\mathrm{p}}$. Hence, the annual interest payable $=\frac{I_{\mathrm{p}} C D}{T} \int_{M}^{t_{1}}\left(\frac{e^{\theta\left(t_{1}-t\right)}-1}{\theta}\right) d t=$ $\frac{I_{\mathrm{p}} C D}{\theta^{2} T}\left[e^{\theta\left(t_{1}-M\right)}-\theta\left(t_{1}-M\right)-1\right]$.

Furthermore, we assume that during the time 0 through $M$, when the account is not settled, the retailer sells the goods and continues to accumulate sales revenue and earns the interest with rate $I_{\mathrm{e}}$. Hence, in this case, the annual interest earned starts from 0 to $M$ and is given by $\frac{I_{\mathrm{e}} P D}{T} \int_{0}^{M} t d t=\frac{I_{\mathrm{e}} P D M^{2}}{2 T}$.

Case ii $t_{w} \leq t_{1} \leq M$

In this sub-case, no interest payable is charged by the retailer, but the retailer earns interest on average 
sales revenues received. Therefore, the annual interest payable $=0$ and the annual interest earned $=$ $\frac{I_{\mathrm{e}} P D}{T}\left[\int_{0}^{t_{1}} t d t+\int_{0}^{t_{1}}\left(M-t_{1}\right) d t\right]=\frac{I_{\mathrm{e}} P D t_{1}}{T}\left[M-\frac{t_{1}}{2}\right]$.

Case iii $0<t_{1}<t_{w}$

If $t_{1}<t_{w}$, then retailer must borrow the partial payment $(1-\alpha) C Q$ at time 0 to pay the supplier and then pays off the loan from sales revenue at time $\frac{(1-\alpha) C}{P}\left(\frac{e^{\theta_{1}}-1}{\theta}\right)$. Hence, the interest charged on the partial payment is from time 0 to $\frac{(1-\alpha) C}{P}\left(\frac{e^{\theta t_{1}}-1}{\theta}\right)$. Thus, the annual interest payable $=\frac{I_{\mathrm{p}} C^{2} D(1-\alpha)^{2}}{2 P T}\left(\frac{e^{\theta t_{1}}-1}{\theta}\right)^{2}$ and the annual interest earned $=\frac{I_{\mathrm{e}} P D}{2 T}\left[t_{1}-\frac{(1-\alpha) C}{P}\left(\frac{e^{\theta t_{1}}-1}{\theta}\right)\right]^{2}+$ $\frac{I_{\mathrm{e}} P D\left(M-t_{1}\right)}{T}\left[t_{1}-\frac{(1-\alpha) C}{P}\left(\frac{e^{\theta t_{1}}-1}{\theta}\right)\right]$.

Thus, from the above arguments, for case I, the total annual cost for the retailer can be expressed as

$T C\left(t_{1}, T\right)= \begin{cases}T C_{1}\left(t_{1}, T\right) & \text { if } M \leq t_{1} \\ T C_{2}\left(t_{1}, T\right) & \text { if } t_{w} \leq t_{1} \leq M \\ T C_{3}\left(t_{1}, T\right) & \text { if } 0<t_{1}<t_{w}\end{cases}$

where

$$
\begin{aligned}
& T C_{1}\left(t_{1}, T\right) \\
& =\frac{D}{T}\left\{\frac{A}{D}+\frac{(h+C \theta)}{\theta^{2}}\left(e^{\theta t_{1}}-\theta t_{1}-1\right)\right. \\
& +\frac{\left(C_{1}+\delta C_{2}\right)}{\delta}\left[\left(T-t_{1}\right)-\frac{\ln \left[1+\delta\left(T-t_{1}\right)\right]}{\delta}\right] \\
& \left.\quad+\frac{I_{\mathrm{p}} C}{\theta^{2}}\left[e^{\theta\left(t_{1}-M\right)}-\theta\left(t_{1}-M\right)-1\right]-\frac{I_{\mathrm{e}} P M^{2}}{2}\right\},
\end{aligned}
$$

$$
\begin{aligned}
& T C_{2}\left(t_{1}, T\right) \\
& =\frac{D}{T}\left\{\frac{A}{D}+\frac{(h+C \theta)}{\theta^{2}}\left(e^{\theta t_{1}}-\theta t_{1}-1\right)\right. \\
& +\frac{\left(C_{1}+\delta C_{2}\right)}{\delta}\left[\left(T-t_{1}\right)-\frac{\ln \left[1+\delta\left(T-t_{1}\right)\right]}{\delta}\right] \\
& \left.-I_{\mathrm{e}} P t_{1}\left[M-\frac{t_{1}}{2}\right]\right\} \text {, }
\end{aligned}
$$

and

$$
\begin{aligned}
T C_{3}\left(t_{1}, T\right) & \\
=\frac{D}{T}\{ & \frac{A}{D}+\frac{(h+C \theta)}{\theta^{2}}\left(e^{\theta t_{1}}-\theta t_{1}-1\right) \\
& +\frac{\left(C_{1}+\delta C_{2}\right)}{\delta}\left[\left(T-t_{1}\right)-\frac{\ln \left[1+\delta\left(T-t_{1}\right)\right]}{\delta}\right] \\
& +\frac{I_{\mathrm{p}}[(1-\alpha) C]^{2}}{2 P}\left(\frac{e^{\theta t_{1}}-1}{\theta}\right)^{2} \\
& -\frac{I_{\mathrm{e}} P}{2}\left[t_{1}-\frac{(1-\alpha) C}{P}\left(\frac{e^{\theta t_{1}}-1}{\theta}\right)\right]^{2} \\
& -I_{\mathrm{e}} P\left(M-t_{1}\right) \\
& \left.\times\left[t_{1}-\frac{(1-\alpha) C}{P}\left(\frac{e^{\theta t_{1}}-1}{\theta}\right)\right]\right\} .
\end{aligned}
$$

Case II $t_{0} \geq t_{w}>M$

Using the similar approach used in case I, in this case the total annual cost for the retailer can be expressed as

$$
T C\left(t_{1}, T\right)= \begin{cases}T C_{1}\left(t_{1}, T\right) & \text { if } t_{w} \leq t_{1} \\ T C_{4}\left(t_{1}, T\right) & \text { if } M \leq t_{1}<t_{w} \\ T C_{3}\left(t_{1}, T\right) & \text { if } t_{1} \leq M\end{cases}
$$

where

$$
\begin{aligned}
& T C_{4}\left(t_{1}, T\right) \\
& \begin{array}{c}
D \\
T
\end{array} \frac{A}{D}+\frac{(h+C \theta)}{\theta^{2}}\left(e^{\theta t_{1}}-\theta t_{1}-1\right) \\
& +\frac{\left(C_{1}+\delta C_{2}\right)}{\delta}\left[\left(T-t_{1}\right)-\frac{\ln \left[1+\delta\left(T-t_{1}\right)\right]}{\delta}\right] \\
& +\frac{I_{\mathrm{p}} C}{\theta^{2}}\left[\left[e^{\theta\left(t_{1}-M\right)}-\theta\left(t_{1}-M\right)-1\right]\right. \\
& \left.+\frac{C\left[(1-\alpha)\left(e^{\theta t_{1}}-1\right)\right]^{2}}{2 P}\right] \\
& \left.-\frac{I_{\mathrm{e}} P}{2}\left[M-\frac{(1-\alpha) C}{P}\left(\frac{e^{\theta t_{1}}-1}{\theta}\right)\right]^{2}\right\}
\end{aligned}
$$


Case III $t_{w}>t_{0}>M$

In this case, if $t_{0} \leq t_{1} \leq t_{w}$, then $M<\frac{(1-\alpha) C}{P}\left(\frac{e^{\theta t_{1}}-1}{\theta}\right)$. Hence, particularly for this sub-case, the retailer must take a loan to pay the supplier the partial payment of $(1-\alpha) C Q$ at time 0 and then take another loan to pay the rest of $\alpha C Q$ at time $M$. The first loan will be paid from the sales revenue received until $\frac{(1-\alpha) C}{P}\left(\frac{e^{\theta t_{1}}-1}{\theta}\right)$. As a result, there is no interest earned. The annual interest payable is

$$
\begin{aligned}
& \frac{I_{\mathrm{p}} C^{2}}{2 P T}(1-\alpha)^{2}\left(\frac{e^{\theta t_{1}}-1}{\theta}\right) Q \\
& +\frac{I_{\mathrm{p}} C \alpha}{T}\left[\frac{(1-\alpha) C}{P}\left(\frac{e^{\theta t_{1}}-1}{\theta}\right)-M\right] Q \\
& +\frac{I_{\mathrm{p}} C^{2} \alpha^{2}}{2 P T}\left(\frac{e^{\theta t_{1}}-1}{\theta}\right) Q \\
& =\frac{I_{\mathrm{p}} C^{2}}{2 P T}\left(1-2 \alpha+2 \alpha^{2}\right)\left(\frac{e^{\theta t_{1}}-1}{\theta}\right) Q \\
& +\frac{I_{\mathrm{p}} C \alpha}{T}\left[\frac{(1-\alpha) C}{P}\left(\frac{e^{\theta t_{1}}-1}{\theta}\right)-M\right] Q
\end{aligned}
$$

For the other sub-cases, we can also obtain the corresponding annual opportunity cost of capital. Therefore, for the retailer in case III, we have the total annual cost as

$$
T C\left(t_{1}, T\right)= \begin{cases}T C_{1}\left(t_{1}, T\right) & \text { if } t_{w} \leq t_{1} \\ T C_{5}\left(t_{1}, T\right) & \text { if } t_{0} \leq t_{1} \leq t_{w} \\ T C_{4}\left(t_{1}, T\right) & \text { if } M \leq t_{1} \leq t_{0} \\ T C_{3}\left(t_{1}, T\right) & \text { if } t_{1} \leq M\end{cases}
$$

where

$$
\begin{aligned}
& T C_{5}\left(t_{1}, T\right) \\
& =\frac{D}{T}\left\{\frac{A}{D}+\frac{h+C \theta}{\theta^{2}}\left(e^{\theta t_{1}}-\theta t_{1}-1\right)\right. \\
& +\frac{\left(C_{1}+\delta C_{2}\right)}{\delta}\left[\left(T-t_{1}\right)-\frac{\ln \left[1+\delta\left(T-t_{1}\right)\right]}{\delta}\right] \\
& +\frac{I_{\mathrm{p}} C\left(1-2 \alpha+2 \alpha^{2}\right)(C / P)}{2}\left(\frac{e^{\theta t_{1}}-1}{\theta}\right)^{2} \\
& +I_{\mathrm{p}} C \alpha\left(\frac{e^{\theta t_{1}}-1}{\theta}\right) \\
& \left.\times\left[(1-\alpha)(C / P)\left(\frac{e^{\theta t_{1}}-1}{\theta}\right)-M\right]\right\}
\end{aligned}
$$

\section{Theoretical results}

Here, we shall determine the optimal shortage point and the optimal replenishment cycle time that minimize the total annual cost for different cases as follows:

Case I $t_{w} \leq t_{1} \leq M$

The necessary conditions for the total annual cost $T C_{1}\left(t_{1}, T\right)$ in Eq. 10 to be minimum are $\frac{\partial T C_{1}\left(t_{1}, T\right)}{\partial t_{1}}=0$ and $\frac{\partial T C_{1}\left(t_{1}, T\right)}{\partial T}=0$ which give

$$
\begin{aligned}
& \frac{\partial T C_{1}\left(t_{1}, T\right)}{\partial t_{1}}=\frac{D}{T}\left\{\frac{(h+C \theta)}{\theta}\left(e^{\theta t_{1}}-1\right)+\frac{\left(C_{1}+\delta C_{2}\right)}{\delta}\right. \\
& \times\left[\frac{1}{1+\delta\left(T-t_{1}\right)}-1\right] \\
& \left.+\frac{I_{\mathrm{p}} C}{\theta}\left(e^{\theta\left(t_{1}-M\right)}-1\right)\right\}=0 \text {, } \\
& \frac{\partial T C_{1}\left(t_{1}, T\right)}{\partial T} \\
& =\frac{D}{T^{2}}\left\{\frac { ( C _ { 1 } + \delta C _ { 2 } ) } { \delta } \left[\frac{\left(T-t_{1}\right)\left(\delta t_{1}-1\right)}{1+\delta\left(T-t_{1}\right)}\right.\right. \\
& \left.+\frac{\ln \left[1+\delta\left(T-t_{1}\right)\right]}{\delta}\right]+\frac{I_{\mathrm{e}} P M^{2}}{2} \\
& -\frac{I_{\mathrm{p}} C}{\theta^{2}}\left[e^{\theta\left(t_{1}-M\right)}-\theta\left(t_{1}-M\right)-1\right] \\
& \left.-\frac{(h+C \theta)}{\theta^{2}}\left(e^{\theta t_{1}}-\theta t_{1}-1\right)-\frac{A}{D}\right\}=0 .
\end{aligned}
$$

For our convenience, let $U=\frac{C_{1}+\delta C_{2}}{\delta}, V=\frac{h+C \theta}{\theta}$, and $W=\frac{I_{\mathrm{p} C}}{\theta}$. Then solving for $T$, from Eq. 18, we have

$$
T=t_{1}+\frac{V\left(e^{\theta t_{1}}-1\right)+W\left(e^{\theta\left(t_{1}-M\right)}-1\right)}{\delta\left[U-V\left(e^{\theta t_{1}}-1\right)-W\left(e^{\theta\left(t_{1}-M\right)}-1\right)\right]}
$$

and Eq. 19 becomes

$$
\begin{aligned}
U & {\left[\frac{\left(T-t_{1}\right)\left(\delta t_{1}-1\right)}{1+\delta\left(T-t_{1}\right)}+\frac{\ln \left[1+\delta\left(T-t_{1}\right)\right]}{\delta}\right] } \\
& +\frac{I_{\mathrm{e}} P M^{2}}{2}-\frac{W}{\theta}\left[e^{\theta\left(t_{1}-M\right)}-\theta\left(t_{1}-M\right)-1\right] \\
& -\frac{V}{\theta}\left(e^{\theta t_{1}}-\theta t_{1}-1\right)-\frac{A}{D}=0 .
\end{aligned}
$$


Substituting Eq. 20 into Eq. 21 yields

$$
\begin{aligned}
& {\left[V\left(e^{\theta t_{1}}-1\right)+W\left(e^{\theta\left(t_{1}-M\right)}-1\right)\right]\left(\frac{\delta t_{1}-1}{\delta}\right)} \\
& +\frac{U}{\delta} \ln \left[\frac{U}{U-V\left(e^{\theta t_{1}}-1\right)+W\left(e^{\theta\left(t_{1}-M\right)}-1\right)}\right] \\
& \quad+\frac{I_{\mathrm{e}} P M^{2}}{2}-\frac{W}{\theta}\left[e^{\theta\left(t_{1}-M\right)}-\theta\left(t_{1}-M\right)-1\right] \\
& \quad-\frac{V}{\theta}\left(e^{\theta t_{1}}-\theta t_{1}-1\right)-\frac{A}{D}=0
\end{aligned}
$$

If we let

$$
\begin{aligned}
\Delta_{1}= & V\left(e^{\theta M}-1\right)\left(\frac{\delta M-1}{\delta}\right) \\
& +\frac{U}{\delta} \ln \left[\frac{U}{U-V\left(e^{\theta M}-1\right)}\right]+\frac{I_{\mathrm{e}} P M^{2}}{2} \\
& -\frac{V}{\theta}\left(e^{\theta M}-\theta M-1\right)-\frac{A}{D}
\end{aligned}
$$

then, we have the following lemma:

\section{Lemma 1}

(a) If $\Delta_{1} \leq 0$, then the total annual cost $T C_{1}\left(t_{1}, T\right)$ has the unique minimum value at $\left(t_{1}, T\right)=\left(t_{11}, T_{1}\right)$ which satisfies Eqs. 20 and 21 where $T_{1} \in[M, \infty)$.

(b) If $\Delta_{1}>0$, then the total annual cost $T C_{1}\left(t_{1}, T\right)$ has a minimum value at $\left(t_{1}, T\right)=\left(M, T_{1}\right)$.

Proof Refer to the "Appendix 1."

Similarly, the necessary conditions for the total annual cost $T C_{2}\left(t_{1}, T\right)$ in Eq. 11 to be minimum are $\frac{\partial T C_{2}\left(t_{1}, T\right)}{\partial t_{1}}=0$ and $\frac{\partial T C_{2}\left(t_{1}, T\right)}{\partial T}=0$ which give

$$
\begin{aligned}
\frac{\partial T C_{2}\left(t_{1}, T\right)}{\partial t_{1}}=\frac{D}{T} & \left\{\frac{(h+C \theta)}{\theta}\left(e^{\theta t_{1}}-1\right)\right. \\
& +\frac{\left(C_{1}+\delta C_{2}\right)}{\delta}\left[\frac{1}{1+\delta\left(T-t_{1}\right)}-1\right] \\
& \left.+I_{\mathrm{e}} P\left(M-t_{1}\right)\right\}=0
\end{aligned}
$$

$$
\begin{aligned}
& \frac{\partial T C_{2}\left(t_{1}, T\right)}{\partial T} \\
& =\frac{D}{T^{2}}\left\{\frac{C_{1}+\delta C_{2}}{\delta}\left[\frac{\left(T-t_{1}\right)\left(\delta t_{1}-1\right)}{1+\delta\left(T-t_{1}\right)}+\frac{\ln \left[1+\delta\left(T-t_{1}\right)\right]}{\delta}\right]\right. \\
& +I_{\mathrm{e}} P\left(M-\frac{t_{1}}{2}\right)-\frac{(h+C \theta)}{\theta^{2}}\left(e^{\theta t_{1}}-\theta t_{1}-1\right) \\
& \left.-\frac{A}{D}\right\}=0 .
\end{aligned}
$$

Again with the help of the same notation, solving for $T$, from Eq. 24, we have

$$
T=t_{1}+\frac{V\left(e^{\theta t_{1}}-1\right)+I_{\mathrm{e}} P\left(M-t_{1}\right)}{\delta\left[U-V\left(e^{\theta t_{1}}-1\right)-I_{\mathrm{e}} P\left(M-t_{1}\right)\right]}
$$

and Eq. 25 becomes

$$
\begin{aligned}
& U\left[\frac{\left(T-t_{1}\right)\left(\delta t_{1}-1\right)}{1+\delta\left(T-t_{1}\right)}+\frac{\ln \left[1+\delta\left(T-t_{1}\right)\right]}{\delta}\right] \\
& +I_{\mathrm{e}} P t_{1}\left[M-\frac{t_{1}}{2}\right]-\frac{V}{\theta}\left(e^{\theta t_{1}}-\theta t_{1}-1\right)-\frac{A}{D}=0 .
\end{aligned}
$$

Substituting Eq. 26 into Eq. 27 yields

$$
\begin{aligned}
& {\left[V\left(e^{\theta t_{1}}-1\right)+I_{\mathrm{e}} P\left(M-t_{1}\right)\right]\left(\frac{\delta t_{1}-1}{\delta}\right)} \\
& \quad+\frac{U}{\delta} \ln \left[\frac{U}{U-V\left(e^{\theta t_{1}}-1\right)-I_{\mathrm{e}} P\left(M-t_{1}\right)}\right] \\
& \quad+I_{\mathrm{e}} P t_{1}\left(M-\frac{t_{1}}{2}\right)-V\left(e^{\theta t_{1}}-\theta t_{1}-1\right)-\frac{A}{D}=0 .
\end{aligned}
$$

Let

$$
\begin{aligned}
\Delta_{2}= & {\left[V\left(e^{\theta t_{w}}-1\right)+I_{\mathrm{e}} P\left(M-t_{1}\right)\right]\left(\frac{\delta t_{w}-1}{\delta}\right) } \\
& +\frac{U}{\delta} \ln \left[\frac{U}{U-V\left(e^{\theta t_{w}}-1\right)+I_{\mathrm{e}} P\left(M-t_{1}\right)}\right] \\
& +I_{\mathrm{e}} P t_{1}\left(M-\frac{t_{w}}{2}\right)-V\left(e^{\theta t_{w}}-\theta t_{w}-1\right)-\frac{A}{D} .
\end{aligned}
$$

It is clear that if $M \geq t_{w}$, then $\Delta_{2} \leq \Delta_{1}$. Then, we have the following lemma:

\section{Lemma 2}

(a) If $\Delta_{2} \leq 0 \leq \Delta_{1}$, then the total annual cost $T C_{2}\left(t_{1}, T\right)$ has the unique minimum value at $\left(t_{1}, T\right)=\left(t_{12}, T_{2}\right)$ which satisfies Eqs. 26 and 27 where $T_{2} \in\left[t_{w}, M\right]$.

(b) If $\Delta_{2}>0$, then the total annual cost $T C_{2}\left(t_{1}, T\right)$ has a minimum value at $\left(t_{1}, T\right)=\left(t_{w}, T_{2}\right)$.

(c) If $\Delta_{1}<0$, then the total annual cost $T C_{2}\left(t_{1}, T\right)$ has a minimum value at $\left(t_{1}, T\right)=\left(M, T_{2}\right)$.

Proof The proof is similar to that in Lemma 1. Hence, we neglect it. 
Again, the necessary conditions for the total annual cost $T C_{3}\left(t_{1}, T\right)$ in Eq. 12 to be minimum are $\frac{\partial T C_{3}\left(t_{1}, T\right)}{\partial t_{1}}=$ 0 and $\frac{\partial T C_{3}\left(t_{1}, T\right)}{\partial T}=0$ which give

$$
\begin{aligned}
& \frac{\partial T C_{3}\left(t_{1}, T\right)}{\partial t_{1}}=\frac{D}{T}\left\{\frac{(h+C \theta)}{\theta}\left(e^{\theta t_{1}}-1\right)\right. \\
&+\frac{\left(C_{1}+\delta C_{2}\right)}{\delta}\left[\frac{1}{1+\delta\left(T-t_{1}\right)}-1\right] \\
&+\frac{I_{\mathrm{p}}[(1-\alpha) C]^{2}}{P}\left(\frac{e^{\theta t_{1}-1}}{\theta}\right) e^{\theta t_{1}} \\
&+I_{\mathrm{e}}(1-\alpha) C e^{\theta t_{1}} \\
& \times\left[\left(t_{1}-\frac{(1-\alpha) C}{P}\left(\frac{e^{\theta t_{1}-1}}{\theta}\right)\right)+1\right] \\
&\left.-I_{\mathrm{e}} P\left(M-t_{1}\right)\right\}=0,
\end{aligned}
$$

$$
\begin{aligned}
& \frac{\partial T C_{3}\left(t_{1}, T\right)}{\partial T} \\
& =\frac{D}{T^{2}}\left\{\frac { ( C _ { 1 } + \delta C _ { 2 } ) } { \delta } \left[\frac{\left(T-t_{1}\right)\left(\delta t_{1}-1\right)}{1+\delta\left(T-t_{1}\right)}\right.\right. \\
& \left.\quad+\frac{\ln \left[1+\delta\left(T-t_{1}\right)\right]}{\delta}\right] \\
& +I_{\mathrm{e}} P\left(M-t_{1}\right)\left[t_{1}-\frac{(1-\alpha) C}{P}\left(\frac{e^{\theta t_{1}}-1}{\theta}\right)\right] \\
& +\frac{I_{\mathrm{e}} P\left[t_{1}-\frac{(1-\alpha) C}{2}\left(\frac{e^{\theta t_{1}}-1}{\theta}\right)^{2}\right]}{} \\
& \quad-\frac{I_{\mathrm{p}} C(1-\alpha)^{2}}{2 P}\left(\frac{e^{\theta t_{1}}-1}{\theta}\right)^{2} \\
& \left.\quad-\frac{(h+C \theta)}{\theta^{2}}\left(e^{\theta t_{1}}-\theta t_{1}-1\right)-\frac{A}{D}\right\}=0 .
\end{aligned}
$$

With the help of the same notation, solving for $T$ from Eq. 30, we have

$$
T=t_{1}+\frac{V\left(e^{\theta t_{1}}-1\right)+W(1-\alpha)^{2}(C / P) e^{\theta t_{1}}\left(e^{\theta t_{1}}-1\right)+I_{\mathrm{e}}(1-\alpha) C e^{\theta t_{1}}\left\{t_{1}-(1-\alpha)(C / P)\left[\left(e^{\theta t_{1}}-1\right) / \theta\right]+1\right\}+I_{\mathrm{e}} P\left(M-t_{1}\right)}{\delta\left\{U-V\left(e^{\theta t_{1}}-1\right)-W(1-\alpha)^{2}(C / P) e^{\theta t_{1}}\left(e^{\theta t_{1}}-1\right)-I_{\mathrm{e}}(1-\alpha) C e^{\theta t_{1}}\left\{t_{1}-(1-\alpha)(C / P)\left[\left(e^{\theta t_{1}}-1\right) / \theta\right]+1\right\}\right\}}
$$

and Eq. 31 becomes

$$
\begin{aligned}
& U\left[\frac{\left(T-t_{1}\right)\left(\delta t_{1}-1\right)}{1+\delta\left(T-t_{1}\right)}+\frac{\ln \left[1+\delta\left(T-t_{1}\right)\right]}{\delta}\right] \\
& +I_{\mathrm{e}} P\left(M-t_{1}\right)\left[t_{1}-\frac{(1-\alpha) C}{P}\left(\frac{e^{\theta t_{1}}-1}{\theta}\right)\right]
\end{aligned}
$$

$$
\begin{aligned}
& +\frac{I_{\mathrm{e}} P}{2}\left[t_{1}-\frac{(1-\alpha) C}{P}\left(\frac{e^{\theta t_{1}}-1}{\theta}\right)^{2}\right] \\
& -\frac{W(1-\alpha)^{2}}{2 \theta P}\left(e^{\theta t_{1}}-1\right)^{2} \\
& -\frac{V}{\theta}\left(e^{\theta t_{1}}-\theta t_{1}-1\right)-\frac{A}{D}=0 .
\end{aligned}
$$

Substituting Eq. 32 into Eq. 33 yields

$$
\begin{aligned}
& {\left[\left[V+W(1-\alpha)^{2}(C / P) e^{\theta t_{1}}\right]\left(e^{\theta t_{1}}-1\right)+I_{\mathrm{e}}(1-\alpha) C e^{\theta t_{1}}\left\{t_{1}-(1-\alpha)(C / P)\left[\left(e^{\theta t_{1}}-1\right) / \theta\right]+1\right\}\right]\left(\frac{\delta t_{1}-1}{\delta}\right)} \\
& +\frac{U}{\delta} \ln \left[\frac{U}{U-\left[V+W(1-\alpha)^{2}(C / P) e^{\theta t_{1}}\right]\left(e^{\theta t_{1}}-1\right)-I_{\mathrm{e}}(1-\alpha) C e^{\theta t_{1}}\left\{t_{1}-(1-\alpha)(C / P)\left[\left(e^{\theta t_{1}}-1\right) / \theta\right]+1\right\}}\right] \\
& +I_{\mathrm{e}} P\left(M-t_{1}\right)\left\{t_{1}-(1-\alpha)(C / P)\left[\left(e^{\theta t_{1}}-1\right) / \theta\right]\right\}+\frac{I_{\mathrm{e}} P}{2}\left\{t_{1}-(1-\alpha)(C / P)\left[\left(e^{\theta t_{1}}-1\right) / \theta\right]^{2}\right\} \\
& -\frac{C}{2} W(1-\alpha)^{2}(C / P)\left[\left(e^{\theta t_{1}}-1\right) / \theta\right]^{2}-\frac{V}{\theta}\left(e^{\theta t_{1}}-\theta t_{1}-1\right)-\frac{A}{D}=0 .
\end{aligned}
$$


If we let

$$
\begin{aligned}
\Delta_{3}= & {\left[\left[V+W(1-\alpha)^{2}(C / P) e^{\theta t_{w}}\right]\left(e^{\theta t_{w}}-1\right)+I_{\mathrm{e}}(1-\alpha) C e^{\theta t_{w}}\left\{t_{w}-(1-\alpha)(C / P)\left[\left(e^{\theta t_{w}}-1\right) / \theta\right]+1\right\}\right]\left(\frac{\delta t_{w}-1}{\delta}\right) } \\
& +\frac{U}{\delta} \ln \left[\frac{U}{U-\left[V+W(1-\alpha)^{2}(C / P) e^{\theta t_{w}}\right]\left(e^{\theta t_{w}}-1\right)-I_{\mathrm{e}}(1-\alpha) C e^{\theta t_{w}}\left\{t_{w}-(1-\alpha)(C / P)\left[\left(e^{\theta t_{w}}-1\right) / \theta\right]+1\right\}}\right] \\
& +I_{\mathrm{e}} P\left(M-t_{w}\right)\left[t_{w}-(1-\alpha)(C / P)\left(e^{\theta t_{w}}-1\right) / \theta\right]+\frac{I_{\mathrm{e}} P}{2}\left\{t_{w}-(1-\alpha)(C / P)\left[\left(e^{\theta t_{w}}-1\right) / \theta\right]^{2}\right\} \\
& -\frac{C}{2} W(1-\alpha)^{2}(C / P)\left[\left(e^{\theta t_{w}}-1\right) / \theta\right]^{2}-\frac{V}{\theta}\left(e^{\theta t_{w}}-\theta t_{w}-1\right)-\frac{A}{D}
\end{aligned}
$$

then, we have the following lemma:

\section{Lemma 3}

(a) If $\Delta_{3} \geq 0$, then the total annual cost $T C_{3}\left(t_{1}, T\right)$ has the unique minimum value at $\left(t_{1}, T\right)=\left(t_{13}, T_{3}\right)$ which satisfies Eqs. 32 and 33 where $T_{3} \in\left(0, t_{w}\right)$.

(b) If $\Delta_{3}<0$, then the value of $T_{3} \in\left(0, t_{w}\right)$ which minimizes $T C_{3}\left(t_{1}, T\right)$ does not exist.

\section{Proof Refer to the "Appendix 2."}

Now, from Eqs. 29 and 35, it is clear that $\Delta_{3} \geq \Delta_{2}$ for $0 \leq \alpha \leq 1$. Moreover, since $M \geq t_{w}$, we come to know that $\Delta_{1} \geq \Delta_{2}$. Consequently, for case I, combining the Lemmas 1-3 and the fact that $T C_{1}\left(t_{1}, M\right)=$ $T C_{2}\left(t_{1}, M\right)$, we can obtain the following theoretical result to determine the optimal shortage point $t_{1}^{*}$ and the optimal cycle time $T^{*}$ :

Theorem 1 For $t_{0}>M \geq t_{w}$, we have

(a) If $\Delta_{1} \leq 0$ and $\Delta_{3}<0$, then $T C\left(t_{1}^{*}, T^{*}\right)=$ $T C_{1}\left(t_{11}, T_{1}\right)$ and $\left(t_{1}^{*}, T^{*}\right)=\left(t_{11}, T_{1}\right)$.

(b) If $\Delta_{1} \leq 0$ and $\Delta_{3} \geq 0$, then $T C\left(t_{1}^{*}, T^{*}\right)=$ $\min \left[T C_{1}\left(t_{11}, T_{1}\right), T C_{3}\left(t_{13}, T_{3}\right)\right]$ and $\left(t_{1}^{*}, T^{*}\right)=$ $\left(t_{11}, T_{1}\right)$ or $\left(t_{13}, T_{3}\right)$ associated with lower cost.

(c) If $\Delta_{1}>0, \Delta_{2}<0$ and $\Delta_{3} \geq 0$, then $T C\left(t_{1}^{*}, T^{*}\right)=$ $\min \left[T C_{2}\left(t_{12}, T_{2}\right), T C_{3}\left(t_{13}, T_{3}\right)\right]$ and $\left(t_{1}^{*}, T^{*}\right)=$ $\left(t_{12}, T_{2}\right)$ or $\left(t_{13}, T_{3}\right)$ associated with lower cost.

(d) If $\Delta_{2} \geq 0$, then $T C\left(t_{1}^{*}, T^{*}\right)=\min \left[T C_{2}\left(t_{w}, T_{2}\right)\right.$, $\left.T C_{3}\left(t_{13}, T_{3}\right)\right]$ and $\left(t_{1}^{*}, T^{*}\right)=\left(t_{w}, T_{2}\right)$ or $\left(t_{13}, T_{3}\right)$ associated with lower cost.

(e) If $\Delta_{1}>0$ and $\Delta_{3}<0$, then $T C\left(t_{1}^{*}, T^{*}\right)=T C_{2}\left(t_{12}\right.$, $\left.T_{2}\right)$ and $\left(t_{1}^{*}, T^{*}\right)=\left(t_{12}, T_{2}\right)$.

Case II $t_{0} \geq t_{w}>M$

Applying the same approach used in case I, the necessary conditions for the total annual cost $T C_{1}\left(t_{1}, T\right)$ in
Eq. 10 to be minimum are the same as Eqs. 20 and 21 . Similarly, to show that there exists a unique value of $\left(t_{1}, T\right)$ in $\left[t_{w}, \infty\right)$ at which $T C_{1}\left(t_{1}, T\right)$ is minimized, we let

$$
\begin{aligned}
\Delta_{4}= & {\left[V\left(e^{\theta t_{w}}-1\right)+W\left(e^{\theta\left(t_{w}-M\right)}-1\right)\right]\left(\frac{\delta t_{w}-1}{\delta}\right) } \\
& +\frac{U}{\delta} \ln \left[\frac{U}{U-V\left(e^{\theta t_{w}}-1\right)-W\left(e^{\theta\left(t_{w}-M\right)}-1\right)}\right] \\
& +\frac{I_{\mathrm{e}} P M^{2}}{2}-\frac{W}{\theta}\left[e^{\theta\left(t_{w}-M\right)}-\theta\left(t_{w}-M\right)-1\right] \\
& -\frac{V}{\theta}\left(e^{\theta t_{w}}-\theta t_{w}-1\right)-\frac{A}{D} .
\end{aligned}
$$

Consequently, we have the following lemma:

\section{Lemma 4}

(a) If $\Delta_{4} \leq 0$, then the total annual cost $T C_{1}\left(t_{1}, T\right)$ has the unique minimum value at $\left(t_{1}, T\right)=\left(t_{11}, T_{1}\right)$ which satisfies Eqs. 20 and 21 where $T_{1} \in\left[t_{w}, \infty\right)$.

(b) If $\Delta_{4}>0$, then the total annual cost $T C_{1}\left(t_{1}, T\right)$ has a minimum value at $\left(t_{1}, T\right)=\left(t_{w}, T_{1}\right)$.

Proof The proof is similar to that in Lemma 1. Hence, we neglect it.

Similarly, the necessary conditions for the total annual cost $T C_{4}\left(t_{1}, T\right)$ in Eq. 14 to be minimum are $\frac{\partial T C_{4}\left(t_{1}, T\right)}{\partial t_{1}}=0$ and $\frac{\partial T C_{4}\left(t_{1}, T\right)}{\partial T}=0$ which give

$$
\begin{aligned}
& \frac{\partial T C_{4}\left(t_{1}, T\right)}{\partial t_{1}} \\
& =\frac{D}{T}\left\{\frac{(h+C \theta)}{\theta}\left(e^{\theta t_{1}}-1\right)+\frac{\left(C_{1}+\delta C_{2}\right)}{\delta}\right. \\
& \times\left[\frac{1}{1+\delta\left(T-t_{1}\right)}-1\right]+\frac{I_{\mathrm{p}} C}{\theta}\left(e^{\theta\left(t_{1}-M\right)}-1\right) \\
& \left.+\left[\frac{I_{\mathrm{p}} C}{\theta}(1-\alpha)+\frac{I_{\mathrm{e}}}{\theta}\right](1-\alpha)(C / P) e^{\theta t_{1}}\left(e^{\theta t_{1}}-1\right)\right\} \\
& =0,
\end{aligned}
$$




$$
\begin{aligned}
& \frac{\partial T C_{4}\left(t_{1}, T\right)}{\partial T} \\
& =\frac{D}{T^{2}}\left\{\frac { ( C _ { 1 } + \delta C _ { 2 } ) } { \delta } \left[\frac{\left(T-t_{1}\right)\left(\delta t_{1}-1\right)}{1+\delta\left(T-t_{1}\right)}\right.\right. \\
& \left.+\frac{\ln \left[1+\delta\left(T-t_{1}\right)\right]}{\delta}\right] \\
& +\frac{I_{\mathrm{e}} P}{2}\left\{\left[M-[(1-\alpha)(C / P)]\left[\left(e^{\theta t_{1}}-1\right) / \theta\right]^{2}\right]\right\}
\end{aligned}
$$

$$
\begin{aligned}
& +\frac{I_{\mathrm{p}} C}{\theta^{2}}\left\{\left[e^{\theta\left(t_{1}-M\right)}-\theta\left(t_{1}-M\right)-1\right]\right. \\
& \left.+(C / 2 P)\left[(1-\alpha)\left(e^{\theta t_{1}}-1\right)\right]^{2}\right\} \\
& \left.-\frac{(h+C \theta)}{\theta^{2}}\left(e^{\theta t_{1}}-\theta t_{1}-1\right)-\frac{A}{D}\right\} .
\end{aligned}
$$

With the help of the same notation, solving for $T$, from Eq. 37, we have

$$
T=t_{1}+\frac{V\left(e^{\theta t_{1}}-1\right)+W\left[e^{\theta\left(t_{1}-M\right)}-1\right]+\left[(1-\alpha) W+\left(I_{\mathrm{e}} / \theta\right)\right](1-\alpha)(C / P) e^{\theta t_{1}}\left(e^{\theta t_{1}}-1\right)}{\delta\left[U-V\left(e^{\theta t_{1}}-1\right)-W\left[e^{\theta\left(t_{1}-M\right)}-1\right]-\left[(1-\alpha) W+\left(I_{\mathrm{e}} / \theta\right)\right](1-\alpha)(C / P) e^{\theta t_{1}}\left(e^{\theta t_{1}}-1\right)\right]}
$$

and Eq. 38 becomes

$$
\begin{aligned}
& U\left[\frac{\left(T-t_{1}\right)\left(\delta t_{1}-1\right)}{1+\delta\left(T-t_{1}\right)}+\frac{\ln \left[1+\delta\left(T-t_{1}\right)\right]}{\delta}\right] \\
& +\frac{I_{\mathrm{e}} P}{2}\left\{\left[M-[(1-\alpha)(C / P)]\left[\left(e^{\theta t_{1}}-1\right) / \theta\right]^{2}\right\}\right.
\end{aligned}
$$

$$
\begin{gathered}
+\frac{W}{\theta}\left\{\left[e^{\theta\left(t_{1}-M\right)}-\theta\left(t_{1}-M\right)-1\right]\right. \\
\left.+(C / 2 P)\left[(1-\alpha)\left(e^{\theta t_{1}}-1\right)\right]^{2}\right\} \\
-\frac{V}{\theta}\left(e^{\theta t_{1}}-\theta t_{1}-1\right)-\frac{A}{D}=0 .
\end{gathered}
$$

Substituting Eq. 39 into Eq. 40 yields

$$
\begin{aligned}
& {\left[V\left(e^{\theta t_{1}}-1\right)+W\left[e^{\theta\left(t_{1}-M\right)}-1\right]+\left[(1-\alpha) W+\left(I_{\mathrm{e}} / \theta\right)\right](1-\alpha) C e^{\theta t_{1}}\left(e^{\theta t_{1}}-1\right)\right]\left(\frac{\delta t_{1}-1}{\delta}\right)} \\
& +\frac{U}{\delta} \ln \left[\frac{U}{U-V\left(e^{\theta t_{1}}-1\right)-W\left[e^{\theta\left(t_{1}-M\right)}-1\right]-\left[(1-\alpha) W+\left(I_{\mathrm{e}} / \theta\right)\right](1-\alpha) C e^{\theta t_{1}}\left(e^{\theta t_{1}}-1\right)}\right] \\
& \left.+\frac{I_{\mathrm{e}} P}{2}\left\{M-[(1-\alpha)(C / P)]\left[\left(e^{\theta t_{1}}-1\right) / \theta\right]^{2}\right\}+\frac{W}{\theta}\left[e^{\theta\left(t_{1}-M\right)}-\theta\left(t_{1}-M\right)-1\right]+(C / 2 P)\left[(1-\alpha)\left(e^{\theta t_{1}}-1\right)\right]^{2}\right] \\
& -\frac{V}{\theta}\left(e^{\theta t_{1}}-\theta t_{1}-1\right)-\frac{A}{D}=0 .
\end{aligned}
$$

To show that there exists a unique value of $\left(t_{1}, T\right)$ in $\left[M, t_{w}\right)$ at which $T C_{4}\left(t_{1}, T\right)$ is minimized, we let

$$
\begin{aligned}
\Delta_{5}= & {\left[V\left(e^{\theta M}-1\right)+\left[(1-\alpha) W+\left(I_{\mathrm{e}} / \theta\right)\right](1-\alpha) C e^{\theta M}\left(e^{\theta M}-1\right)\right]\left(\frac{\delta M-1}{\delta}\right) } \\
& +\frac{U}{\delta} \ln \left[\frac{U}{U-V\left(e^{\theta M}-1\right)-\left[(1-\alpha) W+\left(I_{\mathrm{e}} / \theta\right)\right](1-\alpha) C e^{\theta M}\left(e^{\theta M}-1\right)}\right] \\
& +\frac{I_{\mathrm{e}} P}{2}\left\{M-[(1-\alpha)(C / P)]\left[\left(e^{\theta M}-1\right) / \theta\right]^{2}\right\}+(C / 2 P)\left[(1-\alpha)\left(e^{\theta M}-1\right)\right]^{2} \\
& -\frac{V}{\theta}\left(e^{\theta M}-\theta M-1\right)-\frac{A}{D},
\end{aligned}
$$


and

$$
\begin{aligned}
\Delta_{6}= & {\left[V\left(e^{\theta t_{w}}-1\right)+\left[(1-\alpha) W+\left(I_{\mathrm{e}} / \theta\right)\right](1-\alpha) C e^{\theta t_{w}}\left(e^{\theta t_{w}-1}\right)\right]\left(\frac{\delta t_{w}-1}{\delta}\right) } \\
& +\frac{U}{\delta} \ln \left[\frac{U}{U-V\left(e^{\theta t_{w}}-1\right)-\left[(1-\alpha) t_{w}+\left(I_{\mathrm{e}} / \theta\right)\right](1-\alpha) C e^{\theta t_{w}}\left(e^{\theta t_{w}}-1\right)}\right] \\
& +\frac{I_{\mathrm{e}} P}{2}\left\{t_{w}-[(1-\alpha)(C / P)]\left[\left(e^{\theta t_{w}}-1\right) / \theta\right]^{2}\right\}+(C / 2 P)\left[(1-\alpha)\left(e^{\theta t_{w}}-1\right)\right]^{2} \\
& -\frac{V}{\theta}\left(e^{\theta t_{w}}-\theta t_{w}-1\right)-\frac{A}{D} .
\end{aligned}
$$

Then, we have the following lemma:

\section{Lemma 5}

(a) If $\Delta_{5} \leq 0 \leq \Delta_{6}$, then the total annual cost $T C_{4}\left(t_{1}, T\right)$ has the unique minimum value at $\left(t_{1}, T\right)=\left(t_{14}, T_{4}\right)$ which satisfies Eqs. 39 and 40 where $T_{4} \in\left[M, t_{w}\right)$.

(b) If $\Delta_{5}>0$, then the total annual cost $T C_{4}\left(t_{1}, T\right)$ has a minimum value at $\left(t_{1}, T\right)=\left(M, T_{4}\right)$.

(c) If $\Delta_{6}<0$, then the value of $T \in\left[M, t_{w}\right)$ which minimizes $T C_{4}\left(t_{1}, T\right)$ does not exist.

Proof The proof of either (a) or (b) is similar to that in Lemma 1. As to (c), it is similar to that in Lemma 3(b).

Since the necessary conditions for the total annual cost $T C_{3}\left(t_{1}, T\right)$ in Eq. 12 to be minimum are the same as Eqs. 32 and 33, we have the following lemma:

\section{Lemma 6}

(a) If $\Delta_{5} \geq 0$, then the total annual cost $T C_{3}\left(t_{1}, T\right)$ has the unique minimum value at $\left(t_{1}, T\right)=\left(t_{13}, T_{3}\right)$ which satisfies Eqs. 32 and 33 where $T_{3} \in(0, M]$.

(b) If $\Delta_{5}<0$, then the total annual cost $T C_{3}\left(t_{1}, T\right)$ has a minimum value at $\left(t_{1}, T\right)=\left(M, T_{3}\right)$.

Proof We neglect the proof because it is similar to that in Lemma 1.

Now, from Eqs. 36 and 43, it is clear that $\Delta_{6} \geq$ $\Delta_{4}$ for $0 \leq \alpha \leq 1$. Moreover, since $M<t_{w}$, we come to know that $\Delta_{6} \geq \Delta_{5}$. Consequently, for case II, combining the Lemmas 4-6 and the fact that $T C_{4}\left(M, T_{4}\right)=$ $T C_{3}\left(M, T_{3}\right)$, we can obtain the following theoretical result to determine the optimal shortage point $t_{1}^{*}$ and the optimal cycle time $T^{*}$ :

Theorem 2 For $t_{0} \geq t_{w}>M$, we have

(a) If $\Delta_{6}<0$, then $T C\left(t_{1}^{*}, T^{*}\right)=\min \left[T C_{1}\left(t_{11}, T_{1}\right)\right.$, $\left.T C_{3}\left(M, T_{3}\right)\right]$ and $\left(t_{1}^{*}, T^{*}\right)=\left(t_{11}, T_{1}\right)$ or $\left(M, T_{3}\right)$ associated with lower cost.

(b) If $\Delta_{4}<0, \Delta_{5}<0$, and $\Delta_{5} \geq 0$, then $T C\left(t_{1}^{*}, T^{*}\right)=$ $\min \left[T C_{1}\left(t_{11}, T_{1}\right), T C_{4}\left(t_{14}, T_{4}\right)\right]$ and $\left(t_{1}^{*}, T^{*}\right)=$ $\left(t_{11}, T_{1}\right)$ or $\left(t_{14}, T_{4}\right)$ associated with lower cost.

(c) If $\Delta_{4}<0$ and $\Delta_{5} \geq 0$, then $T C\left(t_{1}^{*}, T^{*}\right)=$ $\min \left[T C_{1}\left(t_{11}, T_{1}\right), T C_{3}\left(t_{13}, T_{3}\right)\right]$ and $\left(t_{1}^{*}, T^{*}\right)=$ $\left(t_{11}, T_{1}\right)$ or $\left(t_{13}, T_{3}\right)$ associated with lower cost.

(d) If $\Delta_{4} \geq 0$ and $\Delta_{5}<0$, then $T C\left(t_{1}^{*}, T^{*}\right)=$ $\min \left[T C_{1}\left(t_{w}, T_{1}\right), T C_{4}\left(t_{14}, T_{4}\right)\right]$ and $\left(t_{1}^{*}, T^{*}\right)=$ $\left(t_{w}, T_{1}\right)$ or $\left(t_{14}, T_{4}\right)$ associated with lower cost.

(e) If $\Delta_{4} \geq 0$ and $\Delta_{5} \geq 0$, then $T C\left(t_{1}^{*}, T^{*}\right)=$ $\min \left[T C_{1}\left(t_{w}, T_{1}\right), T C_{3}\left(t_{13}, T_{3}\right)\right]$ and $\left(t_{1}^{*}, T^{*}\right)=$ $\left(t_{w}, T_{1}\right)$ or $\left(t_{13}, T_{3}\right)$ associated with lower cost.

Case III $t_{w}>t_{0}>M$

From Lemma 4, we come to know that if $\Delta_{4} \leq 0$, then the total annual cost $T C_{1}\left(t_{1}, T\right)$ in Eq. 10 has the unique minimum value at $\left(t_{11}, T_{1}\right)$, where $T_{1} \in\left[t_{w}, \infty\right)$ and satisfies Eqs. 20 and 21. Otherwise, $T C_{1}\left(t_{1}, T\right)$ has minimum value at $\left(t_{w}, T_{1}\right)$. 
Now, the necessary conditions for the total annual cost $T C_{5}\left(t_{1}, T\right)$ in Eq. 17 to be minimum are $\frac{\partial T C_{5}\left(t_{1}, T\right)}{\partial t_{1}}=$ 0 and $\frac{\partial T C_{5}\left(t_{1}, T\right)}{\partial T}=0$ which give

$$
\begin{aligned}
& \frac{\partial T C_{5}\left(t_{1}, T\right)}{\partial t_{1}} \\
& =\frac{D}{T}\left\{V\left(e^{\theta t_{1}}-1\right)+U\left(\frac{1}{1+\delta\left(T-t_{1}\right)}-1\right)\right. \\
& \left.\quad+W e^{\theta t_{1}}\left[(C / P)\left(e^{\theta t_{1}}-1\right)-M \alpha \theta\right]\right\}=0,
\end{aligned}
$$

$$
\begin{aligned}
& \frac{\partial T C_{5}\left(t_{1}, T\right)}{\partial T} \\
& =\frac{D}{T^{2}}\left\{U\left[\frac{\left(T-t_{1}\right)\left(\delta t_{1}-1\right)}{1+\delta\left(T-t_{1}\right)}+\frac{\ln \left[1+\delta\left(T-t_{1}\right)\right]}{\delta}\right]\right. \\
& -W \alpha\left(e^{\theta t_{1}}-1\right) \\
& \times\left[(1-\alpha)(C / P)\left[\left(e^{\theta t_{1}}-1\right) / \theta\right]-M\right]
\end{aligned}
$$

$$
\begin{aligned}
& -\frac{W}{2}\left(1-2 \alpha+2 \alpha^{2}\right)(C / P)\left(e^{\theta t_{1}}-1\right)^{2} \\
& \left.-\frac{V}{\theta}\left(e^{\theta t_{1}}-\theta t_{1}-1\right)-\frac{A}{D}\right\}=0 .
\end{aligned}
$$

With the help of the same notation, solving for $T$, from Eq. 44, we have

$$
T=t_{1}+\frac{V\left(e^{\theta t_{1}}-1\right)+W e^{\theta t_{1}}\left[(C / P)\left(e^{\theta t_{1}}-1\right)-M \alpha \theta\right]}{\delta\left[V\left(e^{\theta t_{1}}-1\right)-W e^{\theta t_{1}}\left[(C / P)\left(e^{\theta t_{1}}-1\right)-M \alpha \theta\right]\right]},
$$

and Eq. 45 becomes

$$
\begin{aligned}
& U\left[\frac{\left(T-t_{1}\right)\left(\delta t_{1}-1\right)}{1+\delta\left(T-t_{1}\right)}+\frac{\ln \left[1+\delta\left(T-t_{1}\right)\right]}{\delta}\right] \\
& -W \alpha\left(e^{\theta t_{1}}-1\right)\left[(1-\alpha)(C / P)\left[\left(e^{\theta t_{1}}-1\right) / \theta\right]-M\right] \\
& -\frac{W}{2}\left(1-2 \alpha+2 \alpha^{2}\right)(C / P)\left(e^{\theta t_{1}}-1\right)^{2} \\
& -\frac{V}{\theta}\left(e^{\theta t_{1}}-\theta t_{1}-1\right)-\frac{A}{D}=0 .
\end{aligned}
$$

Substituting Eq. 46 into Eq. 47 yields

$$
\begin{aligned}
& {\left[V\left(e^{\theta t_{1}}-1\right)+W\left(e^{\theta\left(t_{1}-M\right)}-1\right)+e^{\theta t_{1}}\left[\left(e^{\theta t_{1}}-1\right) / \theta\right](1-\alpha) C(1-\alpha)(C / P)+I_{\mathrm{e}}\right]\left(\frac{\delta t_{1}-1}{\delta}\right)} \\
& +\frac{U}{\delta} \ln \left[\frac{U}{U-V\left(e^{\theta t_{1}}-1\right)-W\left[e^{\theta\left(t_{1}-M\right)}-1\right]-e^{\theta t_{1}}\left[\left(e^{\theta t_{1}}-1\right) / \theta\right](1-\alpha) C\left[(1-\alpha)(C / P)+I_{\mathrm{e}}\right]}\right] \\
& -\frac{W}{2}\left(1-2 \alpha+2 \alpha^{2}\right)(C / P)\left(e^{\theta t_{1}}-1\right)^{2}-\frac{V}{\theta}\left(e^{\theta t_{1}}-\theta t_{1}-1\right)-\frac{A}{D}=0 .
\end{aligned}
$$

To show that there exists a unique value of $\left(t_{1}, T\right)$ in $\left[t_{0}, t_{w}\right)$ at which $T C_{5}\left(t_{1}, T\right)$ is minimized, we let

$$
\begin{aligned}
\Delta_{7}= & {\left[V\left(e^{\theta t_{0}}-1\right)+W\left[e^{\theta\left(t_{0}-M\right)}-1\right]+e^{\theta t_{0}}\left[\left(e^{\theta t_{0}}-1\right) / \theta\right](1-\alpha) C\left[(1-\alpha)(C / P)+I_{\mathrm{e}}\right]\right]\left(\frac{\delta t_{0}-1}{\delta}\right) } \\
& +\frac{U}{\delta} \ln \left[\frac{U}{\left.U-V\left(e^{\theta t_{0}}-1\right)-W\left[e^{\theta\left(t_{0}-M\right)}-1\right]-e^{\theta t_{0}}\left[\left(e^{\theta t_{0}}-1\right) / \theta\right](1-\alpha) C\left[(1-\alpha)(C / P)+I_{\mathrm{e}}\right]\right)}\right] \\
& -\frac{W}{2}\left(1-2 \alpha+2 \alpha^{2}\right)(C / P)\left(e^{\theta t_{0}}-1\right)^{2}-\frac{V}{\theta}\left(e^{\theta t_{0}}-\theta t_{0}-1\right)-\frac{A}{D}=0,
\end{aligned}
$$

and

$$
\begin{aligned}
\Delta_{8}= & {\left[V\left(e^{\theta t_{w}}-1\right)+W\left[e^{\theta\left(t_{w}-M\right)}-1\right]+e^{\theta t_{w}}\left[\left(e^{\theta t_{w}}-1\right) / \theta\right](1-\alpha) C\left[(1-\alpha)(C / P)+I_{\mathrm{e}}\right]\right]\left(\frac{\delta t_{w}-1}{\delta}\right) } \\
& +\frac{U}{\delta} \ln \left[\frac{U}{U-V\left(e^{\theta t_{w}}-1\right)-W\left[e^{\theta\left(t_{w}-M\right)}-1\right]-e^{\theta t_{w}}\left[\left(e^{\theta t_{w}}-1\right) / \theta\right](1-\alpha) C\left[(1-\alpha)(C / P)+I_{\mathrm{e}}\right]}\right] \\
& -\frac{W}{2}\left(1-2 \alpha+2 \alpha^{2}\right)(C / P)\left(e^{\theta t_{w}}-1\right)^{2}-\frac{V}{\theta}\left(e^{\theta t_{w}}-\theta t_{w}-1\right)-\frac{A}{D} .
\end{aligned}
$$


Consequently, we have the following lemma:

\section{Lemma 7}

(a) If $\Delta_{7} \leq 0 \leq \Delta_{8}$, then the total annual cost $T C_{5}\left(t_{1}, T\right)$ has the unique minimum value at $\left(t_{1}, T\right)=\left(t_{15}, T_{5}\right)$ which satisfies Eqs. 46 and 47 where $T_{5} \in\left[t_{0}, t_{w}\right)$.

(b) If $\Delta_{7}>0$, then the total annual cost $T C_{5}\left(t_{1}, T\right)$ has a minimum value at $\left(t_{1}, T\right)=\left(t_{0}, T_{5}\right)$.

(c) If $\Delta_{8}<0$, then the value of $T \in\left[t_{0}, t_{w}\right)$ which minimizes $T C_{5}\left(t_{1}, T\right)$ does not exist.
Proof The proof of either (a) or (b) is similar to that in Lemma 1. As to (c), it is similar to that in Lemma 3(b).

Again, the necessary conditions for the total cost $T C_{4}\left(t_{1}, T\right)$ in Eq. 14 to be minimum are the same as Eqs. 39 and 40. To show that there exists a unique value of $\left(t_{1}, T\right)$ in $\left[M, t_{0}\right]$ at which $T C_{4}\left(t_{4}, T\right)$ is minimized, we let

$$
\begin{aligned}
\Delta_{9}= & {\left[V\left(e^{\theta t_{0}}-1\right)+W\left[e^{\left(\theta t_{0}-M\right)}-1\right]+\left[(1-\alpha) W+\left(I_{\mathrm{e}} / \theta\right)\right](1-\alpha) C e^{\theta t_{0}}\left(e^{\theta t_{0}}-1\right)\right]\left(\frac{\delta t_{0}-1}{\delta}\right) } \\
& +\frac{U}{\delta} \ln \left[\frac{U}{U-V\left(e^{\theta t_{0}}-1\right)-W\left[e^{\left(\theta t_{0}-M\right)}-1\right]-\left[(1-\alpha) W+\left(I_{\mathrm{e}} / \theta\right)\right](1-\alpha) C e^{\theta t_{0}}\left(e^{\theta t_{0}}-1\right)}\right] \\
& +\frac{I_{\mathrm{e}} P}{2}\left[M-(1-\alpha)(C / P)\left[\left(e^{\theta t_{0}}-1\right) / \theta\right]^{2}\right]+\frac{W}{\theta}\left[\left[e^{\theta\left(t_{0}-M\right)}-\theta\left(t_{0}-M\right)-1\right]+(C / 2)\left[(1-\alpha)\left(e^{\theta t_{0}}-1\right)\right]^{2}\right] \\
& -\frac{V}{\theta}\left(e^{\theta t_{0}}-\theta t_{0}-1\right)-\frac{A}{D}=0 .
\end{aligned}
$$

Then, we have the following lemma:

\section{Lemma 8}

(a) If $\Delta_{5} \leq 0 \leq \Delta_{9}$, then the total annual cost $T C_{4}\left(t_{1}, T\right)$ has the unique minimum value at $\left(t_{1}, T\right)=\left(t_{14}, T_{4}\right)$ which satisfies Eqs. 39 and 40 where $T_{4} \in\left[M, t_{0}\right]$.

(b) If $\Delta_{5}>0$, then the total annual cost $T C_{4}\left(t_{1}, T\right)$ has a minimum value at $\left(t_{1}, T\right)=\left(M, T_{4}\right)$.

(c) If $\Delta_{9}<0$, then the total annual cost $T C_{4}\left(t_{1}, T\right)$ has a minimum value at $\left(t_{1}, T\right)=\left(t_{0}, T_{4}\right)$.

Proof We neglect the proof because it is similar to that in Lemma 1.

From Lemma 6, we come to know that if $\Delta_{5} \geq 0$, then the total annual cost $T C_{3}\left(t_{1}, T_{3}\right)$ in Eq. 12 has the unique minimum value at $\left(t_{13}, T_{3}\right)$, where $T_{3} \in(0, M]$ and satisfies Eqs. 32 and 33. Otherwise, $T C_{3}\left(t_{13}, T_{3}\right)$ has minimum value at $\left(M, T_{3}\right)$.

From Eqs. 49 and 51, it is clear that $\Delta_{9} \geq \Delta_{7}$. Again, since $t_{w}>t_{0}>M$, we come to know that $\Delta_{9} \geq \Delta_{5}$ and $\Delta_{8} \geq \Delta_{7} \geq \Delta_{5}$. Consequently, for case III, combining Lemmas 7 and 8 and the fact that $T C_{4}\left(M, T_{4}\right)=$ $T C_{3}\left(M, T_{3}\right)$, we can obtain the following theorem:
Theorem 3 For $t_{0} \geq t_{w}>M$, we have

(a) If $\Delta_{4}<0, \Delta_{8}<0$, and $\Delta_{9}<0$, then $T C\left(t_{1}^{*}\right.$, $\left.T^{*}\right)=\min \left[T C_{1}\left(t_{11}, T_{1}\right), T C_{4}\left(t_{0}, T_{4}\right)\right]$ and $\left(t_{1}^{*}, T^{*}\right)=$ $\left(t_{11}, T_{1}\right)$ or $\left(t_{0}, T_{4}\right)$ associated with lower cost.

(b) If $\Delta_{4}<0, \Delta_{8}<0$, and $\Delta_{9} \geq 0$, then $T C\left(t_{1}^{*}\right.$, $\left.T^{*}\right)=\min \left[T C_{1}\left(t_{11}, T_{1}\right), T C_{4}\left(t_{14}, T_{4}\right)\right]$ and $\left(t_{1}^{*}, T^{*}\right)=$ $\left(t_{11}, T_{1}\right)$ or $\left(t_{14}, T_{4}\right)$ associated with lower cost.

(c) If $\Delta_{4}<0, \Delta_{8} \geq 0$, and $\Delta_{9} \geq 0$, then $T C\left(t_{1}^{*}, T^{*}\right)=$ $\min \left[T C_{1}\left(t_{11}, T_{1}\right), T C_{4}\left(t_{0}, T_{4}\right), T C_{5}\left(t_{15}, T_{5}\right)\right]$ and $\left(t_{1}^{*}, T^{*}\right)=\left(t_{11}, T_{1}\right)$ or $\left(t_{0}, T_{4}\right)$ or $\left(t_{15}, T_{5}\right)$ associated with lower cost.

(d) If $\Delta_{4}<0, \Delta_{7}<0, \Delta_{8} \geq 0$, and $\Delta_{9} \geq 0$, then $\operatorname{TC}\left(t_{1}^{*}\right.$, $\left.T^{*}\right)=\min \left[T C_{1}\left(t_{11}, T_{1}\right), T C_{4}\left(t_{14}, T_{4}\right), T C_{5}\left(t_{15}, T_{5}\right)\right]$ and $\left(t_{1}^{*}, T^{*}\right)=\left(t_{11}, T_{1}\right)$ or $\left(t_{14}, T_{4}\right)$ or $\left(t_{15}, T_{5}\right)$ associated with lower cost.

(e) If $\Delta_{4}<0, \Delta_{5}<0$, and $\Delta_{7} \geq 0$, then $T C\left(t_{1}^{*}, T^{*}\right)=$ $\min \left[T C_{1}\left(t_{11}, T_{1}\right), T C_{4}\left(t_{14}, T_{4}\right), T C_{5}\left(t_{0}, T_{5}\right)\right]$ and $\left(t_{1}^{*}, T^{*}\right)=\left(t_{11}, T_{1}\right)$ or $\left(t_{14}, T_{4}\right)$ or $\left(t_{0}, T_{5}\right)$ associated with lower cost.

(f) If $\Delta_{4}<0$ and $\Delta_{5} \geq 0$, then $T C\left(t_{1}^{*}, T^{*}\right)=$ $\min \left[T C_{1}\left(t_{11}, T_{1}\right), T C_{3}\left(t_{13}, T_{3}\right), T C_{5}\left(t_{0}, T_{5}\right)\right]$ and $\left(t_{1}^{*}, T^{*}\right)=\left(t_{11}, T_{1}\right)$ or $\left(t_{13}, T_{3}\right)$ or $\left(t_{0}, T_{5}\right)$ associated with lower cost. 
(g) If $\Delta_{4} \geq 0, \Delta_{8}<0$, and $\Delta_{9}<0$, then $T C\left(t_{1}^{*}, T^{*}\right)=\min \left[T C_{1}\left(t_{w}, T_{1}\right), T C_{4}\left(t_{0}, T_{4}\right)\right]$ and $\left(t_{1}^{*}, T^{*}\right)=\left(t_{w}, T_{1}\right)$ or $\left(t_{0}, T_{4}\right)$ associated with lower cost.

(h) If $\Delta_{4} \geq 0, \Delta_{8}<0$, and $\Delta_{9} \geq 0$, then $T C\left(t_{1}^{*}, T^{*}\right)=$ $\min \left[T C_{1}\left(t_{w}, T_{1}\right), T C_{4}\left(t_{14}, T_{4}\right)\right]$ and $\left(t_{11}^{*}, T^{*}\right)=$ $\left(t_{w}, T_{1}\right)$ or $\left(t_{14}, T_{4}\right)$ associated with lower cost.

(i) If $\Delta_{4} \geq 0, \Delta_{8} \geq 0$, and $\Delta_{9} \geq 0$, then $T C\left(t_{1}^{*}, T^{*}\right)=$ $\min \left[T C_{1}\left(t_{w}, T_{1}\right), T C_{4}\left(t_{0}, T_{4}\right), T C_{5}\left(t_{15}, T_{5}\right)\right]$ and $\left(t_{1}^{*}, T^{*}\right)=\left(t_{w}, T_{1}\right)$ or $\left(t_{0}, T_{4}\right)$ or $\left(t_{15}, T_{5}\right)$ associated with lower cost.

(j) If $\Delta_{4} \geq 0, \Delta_{7}<0, \Delta_{8} \geq 0>$, and $>\Delta_{9} \geq 0$, then $T C\left(t_{1}^{*}, T^{*}\right)=\min \left[T C_{1}\left(t_{w}, T_{1}\right), T C_{4}\left(t_{14}, T_{4}\right)\right.$, $T C_{5}\left(t_{15}, T_{5}\right)$ big $]$ and $\left(t_{1}^{*}, T^{*}\right)=\left(t_{w}, T_{1}\right)$ or $\left(t_{14}, T_{4}\right)$ or $\left(t_{15}, T_{5}\right)$ associated with lower cost.

(k) If $\Delta_{4} \geq 0, \Delta_{5}<0$, and $\Delta_{7} \geq 0$, then $T C\left(t_{1}^{*}, T^{*}\right)=$ $\min \left[T C_{1}\left(t_{w}, T_{1}\right), T C_{4}\left(t_{14}, T_{4}\right), T C_{5}\left(t_{0}, T_{5}\right)\right]$ and $\left(t_{1}^{*}, T^{*}\right)=\left(t_{w}, T_{1}\right)$ or $\left(t_{14}, T_{4}\right)$ or $\left(t_{0}, T_{5}\right)$ associated with lower cost.

(l) If $\Delta_{4} \geq 0$ and $\Delta_{5} \geq 0$, then $T C\left(t_{1}^{*}, T^{*}\right)=$ $\min \left[T C_{1}\left(t_{w}, T_{1}\right), T C_{3}\left(t_{13}, T_{3}\right), T C_{5}\left(t_{0}, T_{5}\right)\right]$ and $\left(t_{1}^{*}, T^{*}\right)=\left(t_{w}, T_{1}\right)$ or $\left(t_{13}, T_{3}\right)$ or $\left(t_{0}, T_{5}\right)$ associated with lower cost.

Computational algorithm Combining the above three theorems, we propose a computational algorithm for solving the problem, and this algorithm is based on Ouyang et al. [23] model except that shortage is here allowed. Based on $t_{0}>M$ and the values of $M, t_{w}$ and $t_{0}$, we have three possible sets of sub-problems: (1) $t_{0}>M \geq t_{w}$, (2) $t_{0} \geq t_{w}>M$, and (3) $t_{w}>t_{0}>M$. According to Theorems 1 and 2, there are five sub-cases each in the first two sub-problems, and as per Theorem 3 , there are 12 sub-cases in the last sub-problem.

Summarizing the above arguments and by using the characteristics of Theorems 1-3, we develop the following algorithm to solve the problem:

Step 1. Compare the values of $t_{0}, M$, and $t_{w}$. If $t_{0}>$ $M \geq t_{w}$, then go to step 2. If $t_{0} \geq t_{w}>M$, then go to step 3. Otherwise, if $t_{w}>t_{0}>M$, then go to step 4 .

Step 2. Calculate the values of $\Delta_{1}, \Delta_{2}$, and $\Delta_{3}$ with the help of Eqs. 23, 29, and 35, respectively.

(1) If $\Delta_{1} \leq 0$ and $\Delta_{3}<0$, then $T C\left(t_{1}^{*}, T^{*}\right)=$ $T C_{1}\left(t_{11}, T_{1}\right)$ and $\left(t_{1}^{*}, T^{*}\right)=\left(t_{11}, T_{1}\right)$. Go to step 5 .

(2) If $\Delta_{1} \leq 0$ and $\Delta_{3} \geq 0$, then $T C\left(t_{1}^{*}, T^{*}\right)=$ $\min \left[T C_{1}\left(t_{11}, T_{1}\right), T C_{3}\left(t_{13}, T_{3}\right)\right]$ and $\left(t_{1}^{*}\right.$, $\left.T^{*}\right)=\left(t_{11}, T_{1}\right)$ or $\left(t_{13}, T_{3}\right)$ associated with lower cost. Go to step 5 .
(3) If $\Delta_{1}>0, \Delta_{2}<0$, and $\Delta_{3} \geq 0$, then $T C\left(t_{1}^{*}, T^{*}\right)=\min \left[T C_{2}\left(t_{12}, T_{2}\right), T C_{3}\left(t_{13}\right.\right.$, $\left.\left.T_{3}\right)\right]$ and $\left(t_{1}^{*}, T^{*}\right)=\left(t_{12}, T_{2}\right)$ or $\left(t_{13}, T_{3}\right)$ associated with lower cost. Go to step 5 .

(4) If $\Delta_{2} \geq 0$, then $T C\left(t_{1}^{*}, T^{*}\right)=\min \left[T C_{2}\left(t_{w}\right.\right.$, $\left.\left.T_{3}\right), T C_{3}\left(t_{13}, T_{3}\right)\right]$ and $\left(t_{1}^{*}, T^{*}\right)=\left(t_{w}, T_{3}\right)$ or $\left(t_{13}, T_{3}\right)$ associated with lower cost. Go to step 5 .

(5) If $\Delta_{1}>0$ and $\Delta_{3}<0$, then $T C\left(t_{1}^{*}, T^{*}\right)=$ $T C_{2}\left(t_{12}, T_{2}\right)$ and $\left(t_{1}^{*}, T^{*}\right)=\left(t_{12}, T_{2}\right)$. Go to step 5 .

Step 3. Calculate the values of $\Delta_{4}, \Delta_{5}$, and $\Delta_{6}$ with the help of Eqs. 36, 42, and 43, respectively.

(1) If $\Delta_{6}<0$, then $T C\left(t_{1}^{*}, T^{*}\right)=\min \left[T C_{1}\left(t_{11}\right.\right.$, $\left.\left.T_{1}\right), T C_{3}\left(M, T_{3}\right)\right]$ and $\left(t_{1}^{*}, T^{*}\right)=\left(t_{11}, T_{1}\right)$ or $\left(M, T_{3}\right)$ associated with lower cost. Go to step 5 .

(2) If $\Delta_{4}<0, \Delta_{5}<0$, and $\Delta_{5} \geq 0$, then $T C\left(t_{1}^{*}, T^{*}\right)=\min \left[T C_{1}\left(t_{11}, T_{1}\right), T C_{4}\left(t_{14}\right.\right.$, $\left.\left.T_{4}\right)\right]$ and $\left(t_{1}^{*}, T^{*}\right)=\left(t_{11}, T_{1}\right)$ or $\left(t_{14}, T_{4}\right)$ associated with lower cost. Go to step 5 .

(3) If $\Delta_{4}<0$ and $\Delta_{5} \geq 0$, then $T C\left(t_{1}^{*}, T^{*}\right)=$ $\min \left[T C_{1}\left(t_{11}, T_{1}\right), T C_{3}\left(t_{13}, T_{3}\right)\right]$ and $\left(t_{1}^{*}\right.$, $\left.T^{*}\right)=\left(t_{11}, T_{1}\right)$ or $\left(t_{13}, T_{3}\right)$ associated with lower cost. Go to step 5 .

(4) If $\Delta_{4} \geq 0$ and $\Delta_{5}<0$, then $T C\left(t_{1}^{*}, T^{*}\right)=$ $\min \left[T C_{1}\left(t_{w}, T_{1}\right), T C_{4}\left(t_{14}, T_{4}\right)\right]$ and $\left(t_{1}^{*}\right.$, $\left.T^{*}\right)=\left(t_{w}, T_{1}\right)$ or $\left(t_{14}, T_{4}\right)$ associated with lower cost. Go to step 5 .

(5) If $\Delta_{4} \geq 0$ and $\Delta_{5} \geq 0$, then $T C\left(t_{1}^{*}, T^{*}\right)=$ $\min \left[T C_{1}\left(t_{w}, T_{1}\right), T C_{3}\left(t_{13}, T_{3}\right)\right]$ and $\left(t_{1}^{*}\right.$, $\left.T^{*}\right)=\left(t_{w}, T_{1}\right)$ or $\left(t_{13}, T_{3}\right)$. Go to step 5 .

Step 4. Calculate the values of $\Delta_{4}, \Delta_{5}, \Delta_{7}, \Delta_{8}$, and $\Delta_{9}$ with the help of Eqs. 36, 42, and 49-51, respectively.

(1) If $\Delta_{4}<0, \Delta_{8}<0$, and $\Delta_{9}<0$, then $T C\left(t_{1}^{*}, T^{*}\right)=\min \left[T C_{1}\left(t_{11}, T_{1}\right), T C_{4}\left(t_{0}\right.\right.$, $\left.\left.T_{4}\right)\right]$ and $\left(t_{1}^{*}, T^{*}\right)=\left(t_{11}, T_{1}\right)$ or $\left(t_{0}, T_{4}\right)$ associated with lower cost. Go to step 5 .

(2) If $\Delta_{4}<0, \Delta_{8}<0$, and $\Delta_{9} \geq 0$, then $T C\left(t_{1}^{*}, T^{*}\right)=\min \left[T C_{1}\left(t_{11}, T_{1}\right), T C_{4}\left(t_{14}\right.\right.$, $\left.\left.T_{4}\right)\right]$ and $\left(t_{1}^{*}, T^{*}\right)=\left(t_{11}, T_{1}\right)$ or $\left(t_{14}, T_{4}\right)$ associated with lower cost. Go to step 5 .

(3) If $\Delta_{4}<0, \Delta_{8} \geq 0$, and $\Delta_{9} \geq 0$, then $T C\left(t_{1}^{*}, T^{*}\right)=\min \left[T C_{1}\left(t_{11}, T_{1}\right), T C_{4}\left(t_{0}\right.\right.$, $\left.\left.T_{4}\right), T C_{5}\left(t_{15}, T_{5}\right)\right]$ and $\left(t_{1}^{*}, T^{*}\right)=\left(t_{11}\right.$, $\left.T_{1}\right)$ or $\left(t_{0}, T_{4}\right)$ or $\left(t_{15}, T_{5}\right)$ associated with lower cost. Go to step 5 .

(4) If $\Delta_{4}<0, \Delta_{7}<0, \Delta_{8} \geq 0$, and $\Delta_{9} \geq$ 0 , then $T C\left(t_{1}^{*}, T^{*}\right)=\min \left[T C_{1}\left(t_{11}, T_{1}\right)\right.$, 
$\left.T C_{4}\left(t_{14}, T_{4}\right), \quad T C_{5}\left(t_{15}, T_{5}\right)\right]$ and $\left(t_{1}^{*}\right.$, $\left.T^{*}\right)=\left(t_{11}, T_{1}\right)$ or $\left(t_{14}, T_{4}\right)$ or $\left(t_{15}, T_{5}\right)$ associated with lower cost. Go to step 5 .

(5) If $\Delta_{4}<0, \Delta_{5}<0$, and $\Delta_{7} \geq 0$, then $T C\left(t_{1}^{*}, T^{*}\right)=\min \left[T C_{1}\left(t_{11}, T_{1}\right), T C_{4}\left(t_{14}\right.\right.$, $\left.\left.T_{4}\right), T C_{5}\left(t_{0}, T_{5}\right)\right]$ and $\left(t_{1}^{*}, T^{*}\right)=\left(t_{11}, T_{1}\right)$ or $\left(t_{14}, T_{4}\right)$ or $\left(t_{0}, T_{5}\right)$ associated with lower cost. Go to step 5 .

(6) If $\Delta_{4}<0$ and $\Delta_{5} \geq 0$, then $T C\left(t_{1}^{*}\right.$, $\left.T^{*}\right)=\min \left[T C_{1}\left(t_{11}, T_{1}\right), T C_{3}\left(t_{13}, T_{3}\right)\right.$, $\left.T C_{5}\left(t_{0}, T_{5}\right)\right]$ and $\left(t_{1}^{*}, T^{*}\right)=\left(t_{11}, T_{1}\right)$ or $\left(t_{13}, T_{3}\right)$ or $\left(t_{0}, T_{5}\right)$ associated with lower cost. Go to step 5 .

(7) If $\Delta_{4} \geq 0, \Delta_{8}<0$, and $\Delta_{9}<0$, then $T C\left(t_{1}^{*}, T^{*}\right)=\min \left[T C_{1}\left(t_{w}, T_{1}\right), T C_{4}\left(t_{0}\right.\right.$, $\left.\left.T_{4}\right)\right]$ and $\left(t_{1}^{*}, T^{*}\right)=\left(t_{w}, T_{1}\right)$ or $\left(t_{0}, T_{4}\right)$ associated with lower cost. Go to step 5 .

(8) If $\Delta_{4} \geq 0, \Delta_{8}<0$, and $\Delta_{9} \geq 0$, then $T C\left(t_{1}^{*}, T^{*}\right)=\min \left[T C_{1}\left(t_{w}, T_{1}\right), T C_{4}\left(t_{14}\right.\right.$, $\left.\left.T_{4}\right)\right]$ and $\left(t_{11}^{*}, T^{*}\right)=\left(t_{w}, T_{1}\right)$ or $\left(t_{14}, T_{4}\right)$ associated with lower cost. Go to step 5 .

(9) If $\Delta_{4} \geq 0, \Delta_{8} \geq 0$, and $\Delta_{9} \geq 0$, then $T C\left(t_{1}^{*}, T^{*}\right)=\min \left[T C_{1}\left(t_{w}, T_{1}\right), T C_{4}\left(t_{0}\right.\right.$, $\left.\left.T_{4}\right), T C_{5}\left(t_{15}, T_{5}\right)\right]$ and $\left(t_{1}^{*}, T^{*}\right)=\left(t_{w}\right.$, $\left.T_{1}\right)$ or $\left(t_{0}, T_{4}\right)$ or $\left(t_{15}, T_{5}\right)$ associated with lower cost. Go to step 5 .

(10) If $\Delta_{4} \geq 0, \Delta_{7}<0, \Delta_{8} \geq 0$, and $\Delta_{9} \geq$ 0 , then $T C\left(t_{1}^{*}, T^{*}\right)=\min \left[T C_{1}\left(t_{w}, T_{1}\right)\right.$, $\left.T C_{4}\left(t_{14}, T_{4}\right), T C_{5}\left(t_{15}, T_{5}\right)\right]$ and $\left(t_{1}^{*}, T^{*}\right)=$ $\left(t_{w}, T_{1}\right)$ or $\left(t_{14}, T_{4}\right)$ or $\left(t_{15}, T_{5}\right)$ associated with lower cost. Go to step 5 .

(11) If $\Delta_{4} \geq 0, \Delta_{5}<0$, and $\Delta_{7} \geq 0$, then $T C\left(t_{1}^{*}, T^{*}\right)=\min \left[T C_{1}\left(t_{w}, T_{1}\right), T C_{4}\left(t_{14}\right.\right.$, $\left.\left.T_{4}\right), T C_{5}\left(t_{0}, T_{5}\right)\right]$ and $\left(t_{1}^{*}, T^{*}\right)=\left(t_{w}, T_{1}\right)$ or $\left(t_{14}, T_{4}\right)$ or $\left(t_{0}, T_{5}\right)$ associated with lower cost. Go to step 5 .

(12) If $\Delta_{4} \geq 0$ and $\Delta_{5} \geq 0$, then $T C\left(t_{1}^{*}\right.$, $\left.T^{*}\right)=\min \left[T C_{1}\left(t_{w}, T_{1}\right), T C_{3}\left(t_{13}, T_{3}\right)\right.$, $\left.T C_{5}\left(t_{0}, T_{5}\right)\right]$ and $\left(t_{1}^{*}, T^{*}\right)=\left(t_{w}, T_{1}\right)$ or $\left(t_{13}, T_{3}\right)$ or $\left(t_{0}, T_{5}\right)$ associated with lower cost. Go to step 5 .

Step 5. Stop.

After obtaining the optimal values of $t_{1}$ and $T$ denoted by $t_{1}^{*}$ and $T^{*}$, respectively, the optimal value of order quantity $Q$, denoted by $Q^{*}$, can be obtained from Eq. 8 .

\section{Special cases}

In this section, we discuss some special cases listed below. (a) If we let $\delta \rightarrow \infty$, we have $T \approx t_{1}$. Thus, the model becomes the case without shortages. By the above parametric considerations, the parameters $C_{1}$ and $C_{2}$ do not take place and therefore $C_{1}=$ 0 and $C_{2}=0$. Adopting the above conditions, Eqs. 10-12, 14, and 17 become as follows:

$$
\begin{aligned}
T C_{1}(T)=\frac{D}{T}\left\{\frac{A}{D}+\frac{(h+C \theta)}{\theta^{2}}\left(e^{\theta T}-\theta T-1\right)\right. \\
+\frac{I_{\mathrm{p}} C}{\theta^{2}}\left[e^{\theta(T-M)}-\theta(T-M)-1\right] \\
\left.-\frac{I_{\mathrm{e}} P M^{2}}{2}\right\}
\end{aligned}
$$

$$
\begin{gathered}
T C_{2}(T)=\frac{D}{T}\left\{\frac{A}{D}+\frac{(h+C \theta)}{\theta^{2}}\left(e^{\theta T}-\theta T-1\right)\right. \\
\left.-I_{\mathrm{e}} P T\left[M-\frac{T}{2}\right]\right\}
\end{gathered}
$$

$$
\begin{aligned}
T C_{3}(T)=\frac{D}{T} & \left\{\frac{A}{D}+\frac{(h+C \theta)}{\theta^{2}}\left(e^{\theta T}-\theta T-1\right)\right. \\
& +\frac{I_{\mathrm{p}}[(1-\alpha) C]^{2}}{2 P}\left(\frac{e^{\theta T}-1}{\theta}\right)^{2} \\
& -\frac{I_{\mathrm{e}} P}{2}\left[T-\frac{(1-\alpha) C}{P}\left(\frac{e^{\theta T}-1}{\theta}\right)\right]^{2} \\
& -I_{\mathrm{e}} P(M-T) \\
& \left.\times\left[T-\frac{(1-\alpha) C}{P}\left(\frac{e^{\theta T}-1}{\theta}\right)\right]\right\},
\end{aligned}
$$

$$
\begin{aligned}
T C_{4}(T)=\frac{D}{T}\left\{\frac{A}{D}+\frac{(h+C \theta)}{\theta^{2}}\left(e^{\theta T}-\theta T-1\right)\right. \\
+\frac{I_{\mathrm{p}} C}{\theta^{2}}\left[\left[e^{\theta(T-M)}-\theta(T-M)-1\right]\right. \\
\left.\quad+\frac{C\left[(1-\alpha)\left(e^{\theta T}-1\right)\right]^{2}}{2 P}\right] \\
-\frac{I_{\mathrm{e}} P}{2} \\
\left.\times\left[M-\frac{(1-\alpha) C}{P}\left(\frac{e^{\theta T}-1}{\theta}\right)\right]^{2}\right\},
\end{aligned}
$$




$$
\begin{aligned}
T C_{5}(T)=\frac{D}{T} & \left\{\frac{A}{D}+\frac{(h+C \theta)}{\theta^{2}}\left(e^{\theta T}-\theta T-1\right)\right. \\
& +\frac{I_{\mathrm{p}} C\left(1-2 \alpha+2 \alpha^{2}\right)(C / P)}{2} \\
& \times\left(\frac{e^{\theta T}-1}{\theta}\right)^{2}+I_{\mathrm{p}} C \alpha\left(\frac{e^{\theta T}-1}{\theta}\right) \\
& \left.\times\left[(1-\alpha)(C / P)\left(\frac{e^{\theta T}-1}{\theta}\right)-M\right]\right\} .
\end{aligned}
$$

The Eqs. 52-56 are consistent with Eqs. 9-11, 13 and 16 in Ouyang et al. [23] model.

(b) If we let $\theta \rightarrow 0$, i.e., when the deterioration is ignored, there is no cost due to deteriorated units. Similarly, $\delta \rightarrow \infty$, we have $T \approx t_{1}$. Hence, the model becomes the case without shortages and without deterioration. By the above parametric considerations, we have $\lim _{\theta \rightarrow 0^{+}} t_{1}=0$. Also when $\alpha=1, w=0$ and adopting the above conditions, the model can be reduced to economic ordering quantity model under permissible delay in payments and the result is the same as that in Teng [1] model.

(c) When $\delta \rightarrow \infty, \theta \rightarrow 0, \alpha=1, P=C$, and $w=0$, the model is the same as Goyal [5] model.

(d) When $\delta \rightarrow \infty, \alpha=1, P=C$, and $w=0$, the model becomes the case for deteriorating items and without shortages. Hence, this model is similar to Aggarwal and Jaggi [12] model.

Thus, numerous previous models such as Ouyang et al. [23], Teng [1], Goyal [5], and Aggarwal and Jaggi [12] models can be treated as special cases of our model.

\section{Numerical examples}

In order to illustrate the solution procedure, let us consider an inventory system with the following data: Let $A=\$ 250$ per order, $D=1,000$ units per year, $h=\$ 15$ per unit per year, $M=0.1233, C_{1}=\$ 30$ per unit, $C_{2}=\$ 25$ per unit, $P=\$ 85$ per unit, $I_{\mathrm{p}}=0.15$ per dollar per year, $I_{\mathrm{e}}=0.12$ per dollar per year, $\theta=0.08$, and $\delta=0.56$.

Using the proposed algorithm, we obtained the optimal results for different parameters of $\alpha=0.3,0.6,0.9$; $w=100,150,200$, and $C=15,25,35$ as shown in the Table 1.
Table 1 Optimal solutions under different parametric values

\begin{tabular}{lllllll}
\hline$\alpha$ & $w$ & $C$ & $t_{1}^{*}$ & $T^{*}$ & $Q^{*}$ & $T C\left(t_{1}^{*}, T^{*}\right)$ \\
\hline 0.3 & 100 & 15 & 0.1364 & 0.1823 & 662 & $2,125.41$ \\
& & 25 & 0.1315 & 0.1801 & 659 & $2,170.72$ \\
& & 35 & 0.1228 & 0.1753 & 656 & $2,203.45$ \\
& 150 & 15 & 0.1627 & 0.1814 & 689 & $2,210.54$ \\
& & 25 & 0.1627 & 0.1814 & 688 & $2,260.72$ \\
& & 35 & 0.1627 & 0.1814 & 688 & $2,301.35$ \\
& 200 & 15 & 0.1361 & 0.1810 & 661 & $2,270.51$ \\
& & 25 & 0.1302 & 0.1781 & 657 & $2,298.41$ \\
0.6 & 100 & 35 & 0.1217 & 0.1703 & 650 & $2,341.52$ \\
& & 15 & 0.1364 & 0.1823 & 662 & $2,125.41$ \\
& & 25 & 0.1315 & 0.1801 & 659 & $2,170.72$ \\
& 150 & 15 & 0.1228 & 0.1753 & 656 & $2,203.45$ \\
& & 25 & 0.1358 & 0.1819 & 688 & $2,191.35$ \\
& & 35 & 0.1627 & 0.1814 & 687 & $2,210.51$ \\
& 200 & 15 & 0.1363 & 0.1814 & 687 & $2,251.63$ \\
& & 25 & 0.1311 & 0.1791 & 662 & $2,270.35$ \\
& & 35 & 0.1223 & 0.1724 & 660 & $2,286.72$ \\
0.9 & 100 & 15 & 0.1364 & 0.1823 & 662 & $2,125.41$ \\
& & 25 & 0.1315 & 0.1801 & 659 & $2,170.72$ \\
& & 35 & 0.1228 & 0.1753 & 656 & $2,203.45$ \\
& 150 & 15 & 0.1365 & 0.1821 & 664 & $2,191.35$ \\
& & 25 & 0.1317 & 0.1825 & 663 & $2,281.52$ \\
& & 35 & 0.1228 & 0.1728 & 655 & $2,303.72$ \\
& & 15 & 0.1361 & 0.1820 & 664 & $2,191.35$ \\
& & 25 & 0.1312 & 0.1791 & 663 & $2,281.53$ \\
& & 35 & 0.1221 & 0.1703 & 655 & $2,303.72$ \\
\hline
\end{tabular}

The following inferences can be made from the results in Table 1:

1. If $w$ is sufficiently large, then the retailer will prefer the partially delayed payment to the fully delayed payment. Thus, if $w$ is sufficiently large, then the benefit from the fully delayed payment is less than the inventory cost increase. From the economical point of view, if $w$ is sufficiently large, then the benefit from the fully delayed payment is made to avoid huge inventory holding cost.

2. If $w$ is not large, then the retailer will order more than or equal to $w$ and take the benefit of the fully delayed payment.

3. If $\alpha$ is sufficiently large, then the partially delayed payment is almost the same as the fully delayed payment. Hence, the retailer will order less than $w$ to reduce inventory cost and enjoy almost the same benefit as the fully delayed payment.

4. If the purchasing cost $C$ increases, then the optimal shortage point $t_{1}^{*}$, replenishment cycle time $T^{*}$, and the ordering quantity $Q^{*}$ will be decreasing while the optimal total annual cost $T C^{*}$ will be increasing. 
These results not only provide a valuable reference for decision makers in planning and controlling the inventory but also provide a useful model for many organizations that use the decision rule to minimize their total costs. Our computational results identify that both supplier and retailer take advantages from the trade credit.

This analysis also ascertain that if the supplier could offer a partial permissible delay in payments even if the order quantity is less than predetermined quantity, then the total inventory cost will decrease. The proposed partial trade credit financing strategy can be implemented in the inventory control of the selling items that deteriorate continuously such as fruits, vegetables, and others.

\section{Sensitivity analysis}

In this section, we study the effect of changes in the major parameters of the system on the optimal length of inventory interval with positive inventory $t_{1}^{*}$, the optimal length of order cycle $T^{*}$, the optimal order quantity per cycle $Q^{*}$, and the minimum total annual cost $T C^{*}$, by assuming the values of the parameters $(\alpha, w, C)$ as $\alpha=0.3, w=100$, and $C=15$. A sensitivity analysis is performed by considering the same numerical example, and computed results are shown in Table 2. Based on the computational results shown in Table 2, we obtain the following managerial insights:

1. The optimal length of inventory interval with positive inventory $t_{1}^{*}$, the optimal length of order cycle $T^{*}$, and the optimal order quantity per cycle $Q^{*}$ decrease while the minimum total annual cost $T C^{*}$ increases with increase in the values of the parameters $h$ and $\theta$. It indicates the following managerial phenomena: It is reasonable that when the holding cost increases, the retailer will shorten the cycle time. If the retailer can effectively reduce the deteriorating rate of item by improving equipment of storehouse, the total annual inventory cost will be lowered.

2. The values of $t_{1}^{*}, T^{*}, Q^{*}$, and $T C^{*}$ increase with increase in the values of parameter $A$. This shows that if the ordering cost per order could be reduced effectively, the total annual cost could be improved.

3. Each of $t_{1}^{*}, T^{*}, Q^{*}$, and $T C^{*}$ decreases with an increase in the credit period $M$. It implies that, the longer the credit period is, the shorter the replenishment cycle, the lower the order quantity and the total annual cost will be. From economical point of view, if the supplier provides a permissible delay in
Table 2 Effect of change in various parameters of the inventory model

\begin{tabular}{|c|c|c|c|c|c|}
\hline $\begin{array}{l}\text { Changing } \\
\text { parameters }\end{array}$ & $\begin{array}{l}\text { Change in } \\
\text { parameters }\end{array}$ & $t_{1}^{*}$ & $T^{*}$ & $Q^{*}$ & $T C\left(t_{1}^{*}, T^{*}\right)$ \\
\hline \multirow[t]{4}{*}{$h$} & 15 & 0.1364 & 0.1823 & 662 & $2,125.41$ \\
\hline & 20 & 0.1358 & 0.1801 & 661 & $2,130.34$ \\
\hline & 25 & 0.1301 & 0.1783 & 660 & $2,136.52$ \\
\hline & 30 & 0.1257 & 0.1766 & 660 & $2,139.58$ \\
\hline \multirow[t]{4}{*}{$A$} & 250 & 0.1364 & 0.1823 & 662 & $2,125.41$ \\
\hline & 300 & 0.1368 & 0.1828 & 662 & $2,130.61$ \\
\hline & 350 & 0.1371 & 0.1831 & 663 & $2,134.75$ \\
\hline & 400 & 0.1375 & 0.1836 & 663 & $2,140.52$ \\
\hline \multirow[t]{4}{*}{$M$} & 0.1233 & 0.1364 & 0.1823 & 663 & $2,138.34$ \\
\hline & 0.1370 & 0.1362 & 0.1815 & 663 & $2,135.61$ \\
\hline & 0.1569 & 0.1358 & 0.1803 & 662 & $2,128.35$ \\
\hline & 0.1644 & 0.1341 & 0.1798 & 662 & $2,125.41$ \\
\hline \multirow[t]{4}{*}{$\delta$} & 0.38 & 0.1345 & 0.1817 & 663 & $2,210.35$ \\
\hline & 0.44 & 0.1351 & 0.1811 & 663 & $2,216.71$ \\
\hline & 0.50 & 0.1360 & 0.1809 & 662 & $2,220.51$ \\
\hline & 0.56 & 0.1364 & 0.1803 & 662 & $2,225.41$ \\
\hline \multirow[t]{4}{*}{$C_{1}$} & 30 & 0.1364 & 0.1823 & 662 & $2,125.41$ \\
\hline & 40 & 0.1368 & 0.1818 & 662 & $2,136.51$ \\
\hline & 50 & 0.1372 & 0.1813 & 661 & $2,141.58$ \\
\hline & 60 & 0.1378 & 0.1809 & 661 & $2,143.62$ \\
\hline \multirow[t]{4}{*}{$C_{2}$} & 25 & 0.1381 & 0.1810 & 661 & $2,144.54$ \\
\hline & 35 & 0.1376 & 0.1815 & 661 & $2,140.32$ \\
\hline & 45 & 0.1371 & 0.1819 & 662 & $2,137.56$ \\
\hline & 55 & 0.1364 & 0.1823 & 662 & $2,125.41$ \\
\hline \multirow[t]{4}{*}{$\theta$} & 0.02 & 0.1382 & 0.1834 & 663 & $2,115.67$ \\
\hline & 0.04 & 0.1379 & 0.1831 & 663 & $2,118.43$ \\
\hline & 0.06 & 0.1368 & 0.1829 & 662 & $2,121.35$ \\
\hline & 0.08 & 0.1364 & 0.1823 & 662 & $2,125.41$ \\
\hline \multirow[t]{4}{*}{$I_{\mathrm{e}}$} & 0.10 & 0.1369 & 0.1826 & 662 & $2,138.75$ \\
\hline & 0.12 & 0.1364 & 0.1823 & 662 & $2,136.67$ \\
\hline & 0.14 & 0.1360 & 0.1820 & 661 & $2,132.41$ \\
\hline & 0.16 & 0.1351 & 0.1814 & 661 & $2,128.51$ \\
\hline \multirow[t]{4}{*}{$I_{\mathrm{p}}$} & 0.13 & 0.1369 & 0.1829 & 662 & $2,120.51$ \\
\hline & 0.15 & 0.1364 & 0.1823 & 662 & $2,125.41$ \\
\hline & 0.18 & 0.1360 & 0.1819 & 661 & $2,129.67$ \\
\hline & 0.21 & 0.1351 & 0.1815 & 661 & $2,136.52$ \\
\hline
\end{tabular}

payments, the retailer will order lower quantity in order to take the benefits of the permissible delay more frequently.

4. Increasing the backlogging parameter $\delta$ decreases the order quantity $Q^{*}$ and increases the total annual cost $T C^{*}$. It indicates that when shortages are completely backlogged, total annual cost becomes lower. Also it can be found that the replenishment cycle time decreases with an increase in the backlogging parameter $\delta$.

5. With the augment of the shortage cost for backlogged item, $C_{1}, t_{1}^{*}$, and $T C^{*}$ increase but $T^{*}$ and $Q^{*}$ decrease. On the other hand, with the increase 
in the values of the parameter, $C_{2}, t_{1}^{*}$, and $T C^{*}$ decrease but $T^{*}$ and $Q^{*}$ increase.

6. As the interest earned $I_{\mathrm{e}}$ increases, $t_{1}^{*}, T^{*}, Q^{*}$, and $T C^{*}$ are marginally decreased. That is, the length of inventory interval with positive inventory, the length of replenishment cycle, order quantity, and total annual cost decrease with increase in $I_{\mathrm{e}}$. This implies that when the interest earned per dollar is high, the total cost is low.

7. Increase in the interest payable $I_{\mathrm{p}}$ results in a decrease in $t_{1}^{*}, T^{*}$, and $Q^{*}$ where as an increase in $T C^{*}$. That is, the total annual cost increases when the annual interest payable per dollar in stock is high. This shows that when the annual interest payable per dollar in stock is high, the retailer should order less amount of inventory.

\section{Concluding remarks}

In this paper, we extend Ouyang et al. [23] model by allowing shortages and analyze the partial trade credit financing in a supply chain by EOQ-based model for decaying items. We assume that the supplier may offer a partial permissible delay in payments even if the order quantity is less than predetermined quantity. Furthermore, we establish several theoretical results which are given as Lemmas 1-8 and Theorems 1-3 to determine the optimal solution under different conditions. An algorithm using the software Matlab 7.0 is developed to derive the optimal shortage point, cycle time, and ordering quantity in order to minimize the total annual cost. A numerical example is presented to illustrate the procedures of algorithm. Comprehensive sensitivity analysis for the effects of the parameters on the decisions is also offered. Based on the sensitivity analysis, we obtain some managerial insights. The results in the numerical example indicate that the retailer trades off the benefits of full delay in payment against the partial delay in payment and always enjoys the full delay in payment.

There are a number of directions in which this research can be extended. One possible extension stems from the demand. For instance, we may extend the constant demand rate to a more realistic time-varying demand rate that is a function of time, selling price, and others. Another potential direction for future research lies in the generalization of the model. We could generalize the model to allow quantity discounts and inflation rate. Also, it is hoped to further incorporate the proposed model into more realistic assumptions, such as a finite rate of replenishment.
Acknowledgements The authors greatly appreciate the seven anonymous reviewers and the Chief Editor John Davies for their valuable and constructive comments and helpful suggestions, which have led to a significant improvement in the earlier version of the manuscript. This research is supported by University Grants Commission-Special Assistance Programme (UGC-SAP), Department of Mathematics, Gandhigram Rural University, Gandhigram, Tamil Nadu, India.

Open Access This article is distributed under the terms of the Creative Commons Attribution Noncommercial License which permits any noncommercial use, distribution, and reproduction in any medium, provided the original author(s) and source are credited.

\section{Appendix 1: Proof of Lemma 1}

Proof of part (a) Set

$$
\begin{aligned}
F_{1}(x)= & {\left[V\left(e^{\theta x}-1\right)+W\left(e^{\theta(x-M)}-1\right)\right]\left(\frac{\delta x-1}{\delta}\right) } \\
& +\frac{U}{\delta} \ln \left[\frac{U}{U-V\left(e^{\theta x}-1\right)+W\left(e^{\theta(x-M)}-1\right)}\right] \\
& +\frac{I_{\mathrm{e}} P M^{2}}{2}-\frac{W}{\theta}\left[e^{\theta(x-M)}-\theta(x-M)-1\right] \\
& -\frac{V}{\theta}\left(e^{\theta x}-\theta x-1\right)-\frac{A}{D}
\end{aligned}
$$

Taking the first derivative of $F_{1}(x)$ with respect to $x \in[M, \infty)$, we get $\frac{d F_{1}(x)}{d x}>0$. Thus, $F_{1}(x)$ is strictly increasing function with respect to $x \in[M, \infty)$. Furthermore, from Eq. 57, we know that $F_{1}(M)=\Delta_{1}$ and $\lim _{x \rightarrow \infty} F_{1}(x)=+\infty$. Therefore, using intermediate value theorem, there exists a unique $t_{1}$, say, $t_{11}^{*} \in[M, \infty)$ such that $F\left(t_{11}^{*}\right)=0$.

Furthermore, taking the second derivative of $T C_{1}\left(t_{1}, T\right)$ with respect to $t_{1}$ and $T$ and then finding the values of these functions at the point $\left(t_{11}^{*}, T_{1}^{*}\right)$, we obtain

$$
\begin{aligned}
& \frac{\partial^{2} T C_{1}\left(t_{1}, T\right)}{\partial t_{1}^{2}}=\frac{D}{T_{1}^{*}}\left[\theta\left(V e^{\theta t_{11}^{*}}+W e^{\theta\left(t_{11}^{*}-M\right)}\right)\right. \\
&\left.+\frac{C_{1}+\delta C_{2}}{\left[1+\delta\left(T_{1}^{*}-t_{11}^{*}\right)\right]^{2}}\right]>0, \\
& \frac{\partial^{2} T C_{1}\left(t_{1}, T\right)}{\partial t_{1} \partial T}=-\frac{D}{T_{1}^{*}}\left[\frac{C_{1}+\delta C_{2}}{\left[1+\delta\left(T_{1}^{*}-t_{11}^{*}\right)\right]^{2}}\right], \\
& \frac{\partial^{2} T C_{1}\left(t_{1}, T\right)}{\partial T^{2}}=\frac{D}{T_{1}^{*}}\left[\frac{C_{1}+\delta C_{2}}{\left[1+\delta\left(T_{1}^{*}-t_{11}^{*}\right)\right]^{2}}\right]>0
\end{aligned}
$$


and

$$
\begin{aligned}
& \left(\frac{\partial^{2} T C_{1}\left(t_{1}, T\right)}{\partial t_{1}^{2}}\right)\left(\frac{\partial^{2} T C_{1}\left(t_{1}, T\right)}{\partial T^{2}}\right)-\left(\frac{\partial^{2} T C_{1}\left(t_{1}, T\right)}{\partial t_{1} \partial T}\right)^{2} \\
& =\left(\frac{D}{T_{1}^{*}}\right)^{2} \theta\left[V e^{\theta t_{11}^{*}}+W e^{\theta\left(t_{11}^{*}-M\right)}\right] \\
& \quad \times\left[\frac{C_{1}+\delta C_{2}}{\left[1+\delta\left(T_{1}^{*}-t_{11}^{*}\right)\right]^{2}}\right]>0
\end{aligned}
$$

Thus, we can easily see that $t_{11} \in[M, \infty)$ is the unique minimum solution of $T C_{1}\left(t_{1}, T\right)$ denoted by $t_{11}^{*}$. Once we obtain $t_{11}^{*}$, then the value of $T$ denoted by $T_{1}^{*}$ can be found from Eq. 20 and is given by

$$
T_{1}^{*}=t_{11}^{*}+\frac{V\left(e^{\theta t_{11}^{*}}-1\right)+W\left[e^{\theta\left(t_{11}^{*}-M\right)}-1\right]}{\delta\left[U-V\left(e^{\theta t_{11}^{*}}-1\right)-W\left[e^{\theta\left(t_{11}^{*}-M\right)}-1\right]\right]}
$$

Proof of part (b) On the other hand, if $\Delta_{1}>0$, then we know that $F(x)>0$, for all $x \in[M, \infty)$. Thus, $\frac{\partial T C_{1}\left(t_{1}, T\right)}{\partial T}=\frac{D F_{1}\left(t_{1}\right)}{T^{2}}>0$ for all $t_{1} \in[M, \infty)$ which implies that $T C_{1}\left(t_{1}, T\right)$ is a strictly increasing function of $T$. Thus, $T C_{1}\left(t_{1}, T\right)$ has a minimum value when $T$ is minimum. On the other hand, from Eq. 17, we can see that $T$ has a minimum value of $M+\frac{V\left(e^{\theta M}-1\right)}{\delta\left[U-V\left(e^{\theta M}-1\right)\right]}$ as $t_{11}=M$. Therefore, $T C_{1}\left(t_{1}, T\right)$ has minimum value at the point $\left(t_{11}^{*}, T^{*}\right)=\left(M, T_{1}^{*}\right)$, where $t_{11}^{*}=M$ and

$$
T_{1}^{*}=M+\frac{V\left(e^{\theta M}-1\right)}{\delta\left[U-V\left(e^{\theta M}-1\right)\right]}
$$

This completes the proof.

\section{Appendix 2: Proof of Lemma 3}

Proof of part (a) To prove Lemma 3, we set

$$
\begin{aligned}
F_{3}(x)= & {\left[\left[V+W(1-\alpha)^{2}(C / P) e^{\theta x}\right]\left(e^{\theta x}-1\right)+I_{\mathrm{e}}(1-\alpha) C e^{\theta x}\left[x-(1-\alpha)(C / P)\left[\left(e^{\theta x}-1\right) / \theta\right]+1\right]\right]\left(\frac{\delta x-1}{\delta}\right) } \\
& +\frac{U}{\delta} \ln \left[\frac{U}{U-\left[V+W(1-\alpha)^{2}(C / P) e^{\theta x}\right]\left(e^{\theta x}-1\right)-I_{\mathrm{e}}(1-\alpha) C e^{\theta x}\left\{x-(1-\alpha)(C / P)\left[\left(e^{\theta x}-1\right) / \theta\right]+1\right\}}\right] \\
& +I_{\mathrm{e}} P(M-x)\left[x-(1-\alpha)(C / P)\left(e^{\theta x}-1\right) / \theta\right]+\frac{I_{\mathrm{e}} P}{2}\left[x-(1-\alpha)(C / P)\left[\left(e^{\theta x}-1\right) / \theta\right]^{2}\right] \\
& -\frac{C}{2} W(1-\alpha)^{2}(C / P)\left[\left(e^{\theta x}-1\right) / \theta\right]^{2}-\frac{V}{\theta}\left[e^{\theta x}-\theta x-1\right]-\frac{A}{D}
\end{aligned}
$$

Taking the first derivative of $F_{3}(x)$ with respect to $x \in\left(0, t_{w}\right)$, we get $\frac{d F_{3}(x)}{d x}>0$. Thus, $F_{3}(x)$ is strictly increasing function with respect to $x \in\left(0, t_{w}\right)$. Furthermore, from Eq. 58, we know that $F_{3}(M)=\Delta_{3}$ and $\lim _{x \rightarrow 0} F_{3}(x)=-A<0$ and $\lim _{x \rightarrow t_{w}^{-}} F_{3}(x)=\Delta_{3}$. Therefore, if $\lim _{x \rightarrow t_{w}^{-}} F_{3}(x)=\Delta_{3} \geq 0$, then by applying intermediate value theorem, there exists a unique $t_{1}$, say, $t_{13}^{*} \in\left(0, t_{w}\right)$ such that $F\left(t_{13}^{*}\right)=0$.

Now, taking the second derivative of $T C_{3}\left(t_{1}, T\right)$ with respect to $t_{1}$ and $\mathrm{T}$ and then finding the values of these functions at the point $\left(t_{1}, T\right)=\left(t_{13}^{*}, T_{3}^{*}\right)$, we obtain

$$
\left(\frac{\partial^{2} T C_{3}\left(t_{1}, T\right)}{\partial t_{1}^{2}}\right)\left(\frac{\partial^{2} T C_{3}\left(t_{1}, T\right)}{\partial T^{2}}\right)-\left(\frac{\partial^{2} T C_{3}\left(t_{1}, T\right)}{\partial t_{1} \partial T}\right)^{2}>0
$$

Thus, we can easily see that $t_{13} \in\left(0, t_{w}\right)$ is the unique minimum solution of $T C_{3}\left(t_{3}, T\right)$ denoted by $t_{13}^{*}$. Once we obtain $t_{13}^{*}$, then the value of $T$ denoted by $T_{3}^{*}$ can be found from Eq. 32 and is given by

$$
T_{3}^{*}=t_{13}^{*}+\frac{V\left(e^{\theta t_{13}^{*}}-1\right)+W(1-\alpha)^{2}(C / P) e^{\theta t_{13}^{*}}\left(e^{\theta t_{13}^{*}}-1\right)+I_{\mathrm{e}}(1-\alpha) C e^{\theta t_{13}^{*}}\left[t_{13}^{*}-(1-\alpha)(C / P)\left(e^{\theta t_{13}^{*}}-1\right) / \theta+1\right]+I_{\mathrm{e}} P\left(M-t_{13}^{*}\right)}{\delta\left\{U-V\left(e^{\theta t_{13}^{*}}-1\right)-W(1-\alpha)^{2}(C / P) e^{\theta t_{13}^{*}}\left(e^{\theta t_{13}^{*}}-1\right)-I_{\mathrm{e}}(1-\alpha) C e^{\theta t t_{3}^{*}}\left[t_{13}^{*}-(1-\alpha)(C / P)\left[\left(e^{\theta t_{13}^{*}}-1\right) / \theta\right]+1\right]\right\}}
$$

Proof of part (b) However, if $\lim _{x \rightarrow t_{w}^{-}} F_{3}(x)=\Delta_{3}<0$, then $F_{3}(x)<0$ for all $t_{13} \in\left(0, t_{w}\right)$. Consequently, we have $\frac{\partial T C_{3}\left(t_{1}, T\right)}{\partial T}=\frac{D F_{3}\left(t_{1}\right)}{T^{2}}<0$ for all $t_{1} \in\left(0, t_{w}\right)$ which implies that $T C_{3}\left(t_{1}, T\right)$ is a strictly decreasing function of 
$T$ in the open interval $\left(0, t_{w}\right)$. Therefore, we cannot find the value of $T$ in the open interval $\left(0, t_{w}\right)$ that minimizes $T C_{3}\left(t_{1}, T\right)$. This completes the proof.

\section{References}

1. Teng JT (2002) An economic order quantity under conditions of permissible delay in payments. J Oper Res Soc 53:915-918

2. Haley CW, Higgins RC (1973) Inventory policy and trade credit financing. Manage Sci 20:464-471

3. Hwang H, Shinn SW (1997) Retailer's pricing and lot sizing policy for exponentially deteriorating products under the condition of permissible delay in payments. Comput Oper Res 24:539-547

4. Jamal AMM, Sarkar BR, Wang S (1997) Optimal payment time for a retailer under permitted delay of payment by the wholesaler. Int J Prod Econ 66:59-66

5. Goyal SK (1985) Economic ordering quantity under conditions of permissible delay in payments. J Oper Res Soc 36:335-343

6. Abad PL, Jaggi CK (2003) A joint approach for setting unit price and the length of the credit period for a seller when end demand is price sensitive. Int $\mathrm{J}$ Prod Econ 83:115-122

7. Huang YF (2003) Optimal retailer's ordering policies in the EOQ model under trade credit financing. J Oper Res Soc 54:1011-1015

8. Chung KJ, Goyal SK, Huang YF (2005) The optimal inventory policies under permissible delay in payments depending on the ordering quantity. Int J Prod Econ 95:203-213

9. Teng JT, Chang CT, Goyal SK (2005) Optimal pricing and ordering policy under permissible delay in payments. Int $\mathrm{J}$ Prod Econ 97:121-129

10. Song X, Cai X (2006) On optimal payment time for a retailer under permitted delay of payment by the wholesaler. Int J Prod Econ 103:246-251

11. Goyal SK, Teng JT, Chang CT (2007) Optimal ordering policies when the supplier provides a progressive interest scheme. Eur J Oper Res 179(2):404-413
12. Aggarwal SP, Jaggi CK (1995) Ordering policies of deteriorating items under permissible delay in payments. J Oper Res Soc 46:658-662

13. Chang HJ, Hung CH, Dye CY (2001) An inventory model for deteriorating items with linear trend demand under conditions of permissible delay in payment. Prod Plan Control 12:274-282

14. Dye, Chung Y (2002) A deteriorating inventory model with stock dependent rate and partial backlogging under conditions of permissible delay in payments. Opsearch 39:189198

15. Ouyang LY, Teng JT, Chen LH (2006) Optimal ordering policy for deteriorating items with partial backlogging under permissible delay in payments. J Glob Optim 34:245-271

16. Tsao YC, Sheen GJ (2008) Dynamic pricing, promotion and replenishment policies for a deteriorating item under permissible delay in payments. Comput Oper Res 35:3562-3580

17. Jaggi CK, Goyal SK, Goel SK (2008) Retailer's optimal replenishment decisions with credit linked demand under permissible delay in payments. Eur J Oper Res 190(1):130-135

18. Soni H, Shah NH (2008) Optimal ordering policy for stockdependent demand under progressive payment scheme. Eur J Oper Res 184:91-100

19. Teng JT, Chern MS, Yang HL, and Wang YJ (1999) Deterministic lot-size inventory model with shortages and deterioration for fluctuating demand. Oper Res Lett 24:65-72

20. Yang HL, Teng JT, Chern MS (2001) Deterministic inventory lot size models under inflation with shortages and deterioration for fluctuating demand. Nav Res Logist 48(2):144158

21. Papachristos S, Skouri K (2000) An optimal replenishment policy for deteriorating items with time-varying demand and partial exponential type backlogging. Oper Res Lett 27(4):175-184

22. Teng JT, Yang HJ, Ouyang LY (2003) On an EOQ model for deteriorating items with time-varying demand and partial backlogging. J Oper Res Soc 54(4):432-436

23. Ouyang LY, Teng JT, Goyal SK, Yang CT (2009) An economic order quantity model for deteriorating items with partially permissible delay in payments linked to order quantity. Eur J Oper Res 194:418-431 